\title{
Sugar $\gamma$-amino acids as building blocks for the synthesis of cyclic neoglycopeptides
}

\author{
Mylène Richard, ${ }^{\mathrm{a}}$ Julen Ariztia, ${ }^{\mathrm{a}}$ Sandrine Lamandé-Langle, ${ }^{\mathrm{a}}$ Nadia Pellegrini Moïse ${ }^{\mathrm{a}}$ \\ a: Université de Lorraine, CNRS, L2CM, F-5400 Nancy, France \\ *Corresponding author, E-mail address: nadia.pellegrini@univ-lorraine.fr (N. Pellegrini \\ Moïse)
}

Keywords: sugar amino acids, cyclopeptides, $C$-glycosyl lactone, multivalent scaffold

\begin{abstract}
Cyclopeptides have interesting biological properties or find potential applications as templates for anchoring of a wide range of biomolecules or detection dyes. Anomeric sugar $\gamma$-amino acids efficiently obtained by nucleophilic addition of nitronate anion on sugar olefins are usefull building blocks for the preparation of new cyclic glycopeptides. We report here the synthesis of two types of cyclic systems containing $\gamma$-glyco amino acid and $\alpha$-amino acid units. A first example was prepared to demonstrate the feasibility of the cyclization of these original structures. To explore these systems as template for multivalency, $\alpha$-amino acid and sugar amino-acid moieties bearing additional functions were choosen as building blocks for the second macrocyle. Furthermore, we describe and explain some unexpected results obtained during deprotection of these new cyclic glycopeptides.
\end{abstract}


Cyclic peptides are naturally occurring substances exhibiting improved properties compared to the linear ones. Indeed, linear peptides usually show low metabolic stability limiting their use as drugs and display conformational flexibility reducing the strength of the ligand-target binding. ${ }^{1-6}$ Therefore, the development of original cyclopeptide-based therapeutics is currently a growing interest. Some of them like the well-known vancomycin and its derivatives, ${ }^{6,7}$ gramicidin, ${ }^{8,9}$ colistin, ${ }^{10}$ octreotide, ${ }^{11}$ and RGD derivatives ${ }^{12,13}$ exhibit useful biological properties as antibiotics, anticancer and integrin inhibitors. Cyclic peptides also proved to be useful for the design of functional nanotubes from self-assembly. ${ }^{14,15}$ Cyclic peptides or peptidomimetics can be useful in drug design ${ }^{16-21}$ and serve as templates for attaching and assembling a wide variety of ligands. ${ }^{5,22,23}$ Such templates allow the facile and versatile attachment of multiple ligands (multivalency) by suitable functions., ${ }^{5,25}$ One noteworthy example are the Regioselectively Addressable Functionalized Templates (RAFT) developed by Dumy et al. ${ }^{26-28}$ which have found powerful applications in biology and imaging. ${ }^{27,29,30} \mathrm{~A}$ wide range of multivalent (glyco)cyclopeptides and synthetic glycoclusters based on cyclic peptides have been described for their related applications in glycobiology. $5,25,31-34$

Designing and synthetizing new types of biocompatible and functionalizable molecular scaffolds for multivalent presentation are great challenges for synthetic chemists. As unusual amino acids with multiple functionalization points, sugar amino acids are good candidates and can be incorporated into peptidic structures. A few years ago, we developed anomeric sugar $\beta$ - and $\gamma$-amino acids ${ }^{35-37}$ and we integrated them into small mixed peptides, ${ }^{38,39}$ and cyclic glycopeptides. ${ }^{38,40}$

In connection with our research on new carbohydrates-containing peptides, we proposed the construction of original hybrid macrocyclic structures built with sugar $\gamma$-amino acids and $\alpha$ amino acids. Furthermore, to explore the scope of these structures as templates for multivalency, both amino-acid moieties were carefully chosen to provide additional anchoring points. We give in this paper a full account of the synthesis of these original cyclic hybrid glycopeptides. 


\section{Results and discussion}

The nucleophilic addition of a nitronate anion on push-pull anomeric sugar olefins has been previously studied in our group (Scheme 1). ${ }^{35}$ Michael adducts 1 were obtained in excellent yield as a mixture of inseparable nitro esters starting from the corresponding erythroglycosylidene (14/86: $\alpha / \beta$ ratio), the major anomer bearing the nitro group on the less hindered face of the sugar. We have previously demonstrated that both anomers are in equilibrium, likely as a result of opening and closure of the sugar ring. The configuration of the major anomer (methyl carboxylate and isopropylidene on the same face) was established on the basis of ${ }^{1} \mathrm{H}$ NMR spectroscopic data by NOESY experiments on $\alpha / \beta$ mixture and X-ray crystallographic analysis. ${ }^{35}$ Anomeric mixture of nitro esters 1 was treated with lithium hydroxide in $\mathrm{THF} / \mathrm{H}_{2} \mathrm{O}$ to give the corresponding carboxylic acid in very good yield. At this stage, the separation of both anomers could be performed by chromatography and the subsequent steps were carried out on the major isomer. Reduction of the nitro group required medium hydrogen pressure (40 psi) to give the expected amine subsequently protected with a benzyloxycarbonyl group under standard conditions. The $\mathrm{NH}-\mathrm{Cbz}$ protected compound 2 thusly prepared was coupled with H-L-ala-OMe giving 3 in $85 \%$ yield. At this point, either amine or acid terminal functions of $\mathbf{3}$ were deprotected by hydrogenolysis $\left(\mathrm{H}_{2}, \mathrm{Pd} / \mathrm{C}, \mathrm{MeOH}\right)$ or by saponification $\left(\mathrm{LiOH}, \mathrm{THF} / \mathrm{H}_{2} \mathrm{O}\right)$ respectively. Building blocks $\mathbf{5}$ and $\mathbf{4}$ were then coupled using HATU (1-[bis(dimethylamino)methylene]-1H-1,2,3-triazolo[4,5-b]pyridinium 3-oxid hexafluorophosphate) as activating agent and led to the tetrapeptide 6 in $78 \%$ yield. Compound 7 was obtained in $79 \%$ yield by hydrogenolysis of $N$-benzyloxycarbonyl protecting group and subsequent coupling with 4 . Having achieved the synthesis of the linear hexapeptide 7, we next envisioned the cyclization step using classical peptide chemistry. First, saponification of the ester function of 7 with $\mathrm{LiOH}$ in $\mathrm{THF} / \mathrm{MeOH}$ mixture led to the crude carboxylic acid. The $N$-benzyloxycarbonyl group was then removed by catalytic hydrogenolysis using $\mathrm{Pd} / \mathrm{C}$ in $\mathrm{MeOH}$. Without purification, the fully deprotected glycopeptide was treated with HATU as coupling agent in $\mathrm{CH}_{2} \mathrm{Cl}_{2}$ /DMF mixture for 3 days under high dilution conditions $(\mathrm{c}=0.1 \mathrm{mmol} / \mathrm{L})$ to promote macrocyclization over poly(oligo)merization. ${ }^{41}$ After purification, the cyclic glycopeptide 8 was obtained in $42 \%$ yield. The use of other coupling agent (benzotriazol-1-yl-oxytripyrrolidinophosphonium hexafluorophosphate, PyBop) did not significantly improve the yield of $\mathbf{8}$. As expected by the symmetric structure of $\mathbf{8},{ }^{1} \mathrm{H}$ and ${ }^{13} \mathrm{C}$ NMR spectra revealed only one set of signals corresponding to a sugar/alanine unit. Nevertheless, mass spectrometry unambiguously 
confirmed the formation of expected cyclic structure (see SI, Figure S1, see scheme 1 for atom numbering).
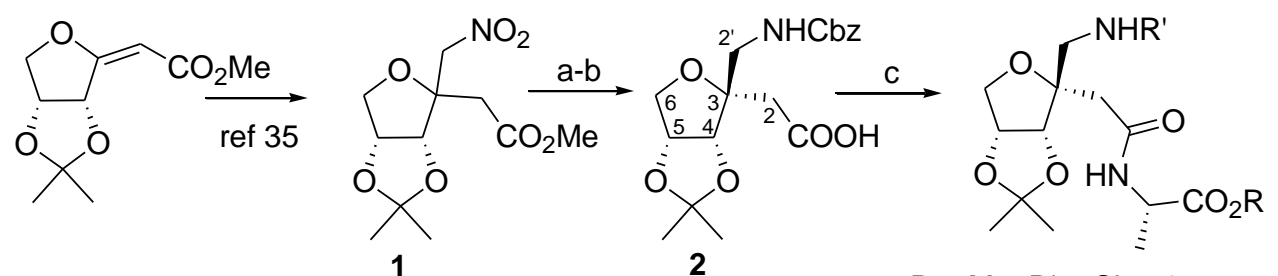

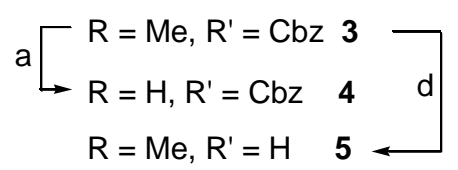

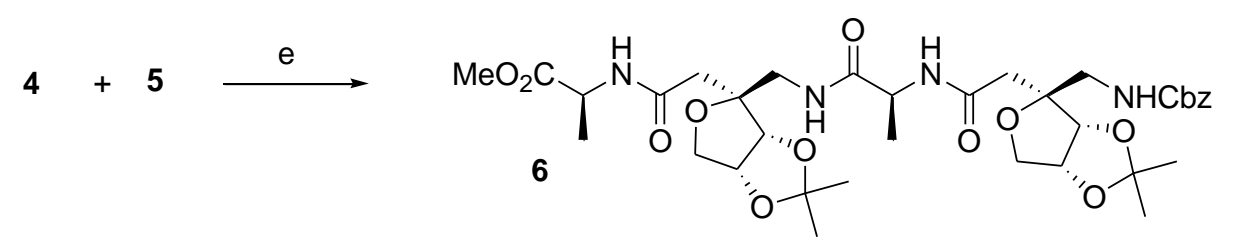

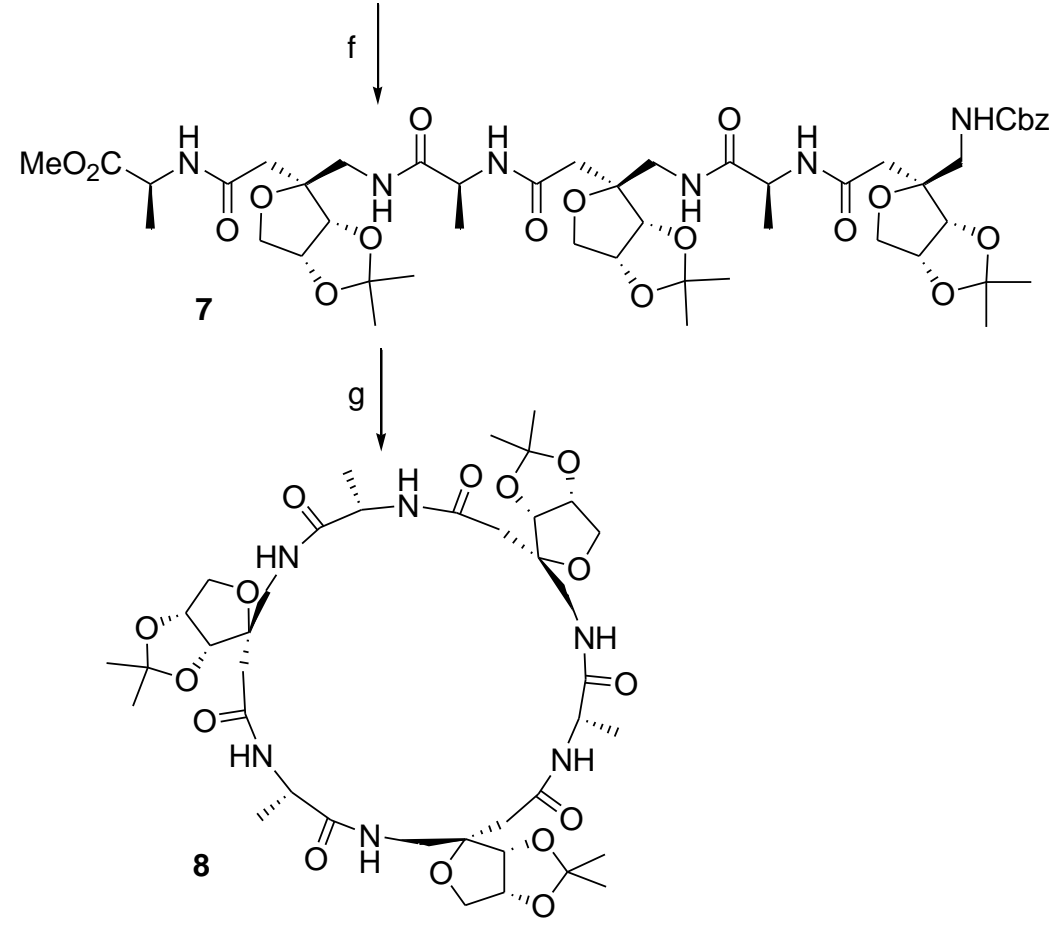

Scheme 1. a: $\mathrm{LiOH}, \mathrm{THF} / \mathrm{H}_{2} \mathrm{O}$ (3/1), 4: quantitative yield; b: 1) $\mathrm{H}_{2}, \mathrm{Pd} / \mathrm{C}(10 \%), \mathrm{MeOH}, 3$ bars, 80\%; 2) TMSCl (2 eq.), DCM, 3) DIEA, CbzCl, 65\% three steps; c: H-L-Ala-OMe, DIEA, HATU, DMF, 85\%; d) $\mathrm{H}_{2}$, Pd/C, MeOH, 99\%; e) DIEA, HATU, DMF, 78\%; f) i. $\mathrm{H}_{2}$, $\mathrm{Pd} / \mathrm{C}, \mathrm{MeOH}$, ii. 4, DIEA, HATU, DMF, 79\%; g) i. $\mathrm{LiOH}, \mathrm{THF} / \mathrm{H}_{2} \mathrm{O}$, ii. $\mathrm{H}_{2}, \mathrm{Pd} / \mathrm{C}, \mathrm{MeOH}$, iii. DIEA, HATU, DMF/CH $\mathrm{Cl}_{2}, 42 \%$. 
After the validation of this proof of concept, i.e. the successful synthesis of $\mathbf{8}$ and the confirmation of the cyclization of these structures, we continued our work with the synthesis of a more functionalized cyclic peptide. This second example is built with $\gamma$-glycoamino acid and $\alpha$-amino acid bearing additional reactive groups (Scheme 2). For this purpose, L-lysine with a tert-butyloxycarbonyl as side chain protecting group was selected as $\alpha$-amino acid partner. Derivative 9 was chosen as $\gamma$-glycoamino acid partner and modified in order to display a hydroxymethyl arm. As explained above, the nitro ester 9 was obtained as a mixture of anomers $(15 / 85 \alpha / \beta$ ratio) by addition of the nitromethane carbanion on the corresponding exo-glycal. The selective removal of the 7,8-O-isopropylidene protecting group was achieved by treatment with $1 \mathrm{~N} \mathrm{HCl}$ in $\mathrm{MeOH}$. The corresponding diol was obtained as a mixture of anomers and separated by column chromatography. ${ }^{35}$ We decided to go ahead with the major isomer $\mathbf{1 0}$ and oxidative cleavage of the diol followed by reduction of the aldehyde intermediate led to compound $\mathbf{1 2}$ bearing a hydroxymethyl arm. The nitro function acting as an amino protecting group was maintained for the first coupling with H-L-Lysine(Boc)-OMe and the compound $\mathbf{1 4}$ was obtained in $90 \%$ yield. We first planned to protect the hydroxyl group as a silyl ether derivative but this protection step appeared unnecessary since the unprotected hydroxyl was not affecting the peptidic coupling. The nitro group was next hydrogenated and the resulting amine $\mathbf{1 5}$ was protected with a benzyloxycarbonyl group. Methyl ester of $\mathbf{1 6}$ could be selectively removed by saponification leading to acid $\mathbf{1 7}$ in quantitative yield. Coupling of compounds 15 and 17 using HATU in DMF led to glycopeptides 18 and then 19 in 69\% and 47\% yields respectively. Cyclic derivative 20 was obtained after amine and acid deprotection followed by activation with HATU and intramolecular coupling in $\mathrm{CH}_{2} \mathrm{Cl}_{2} / \mathrm{DMF}$ mixture under high dilution conditions ( $\mathrm{c}=0.1$ $\mathrm{mmol} / \mathrm{L}$ ). Retrieval of compound $\mathbf{2 0}$ in the crude was tedious and purification inefficient although the expected molecular pic was detected by mass spectrometry $\left([\mathrm{M}+\mathrm{Na}]^{+}=\right.$ 1436.7626, calculated for $\mathrm{C}_{66} \mathrm{H}_{111} \mathrm{~N}_{9} \mathrm{O}_{24} \mathrm{Na}$ : 1436.7634). We next turned to PyBop as activating agent in the same high dilution conditions. In this case, the recovery of $\mathbf{2 0}$ was easier but also required two purifications by column chromatography on silica gel explaining the low yield of $\mathbf{2 0}(22 \%)$. Even though the cyclization step was tedious, we were pleased to obtain a macrocycle bearing two different reactive groups. 

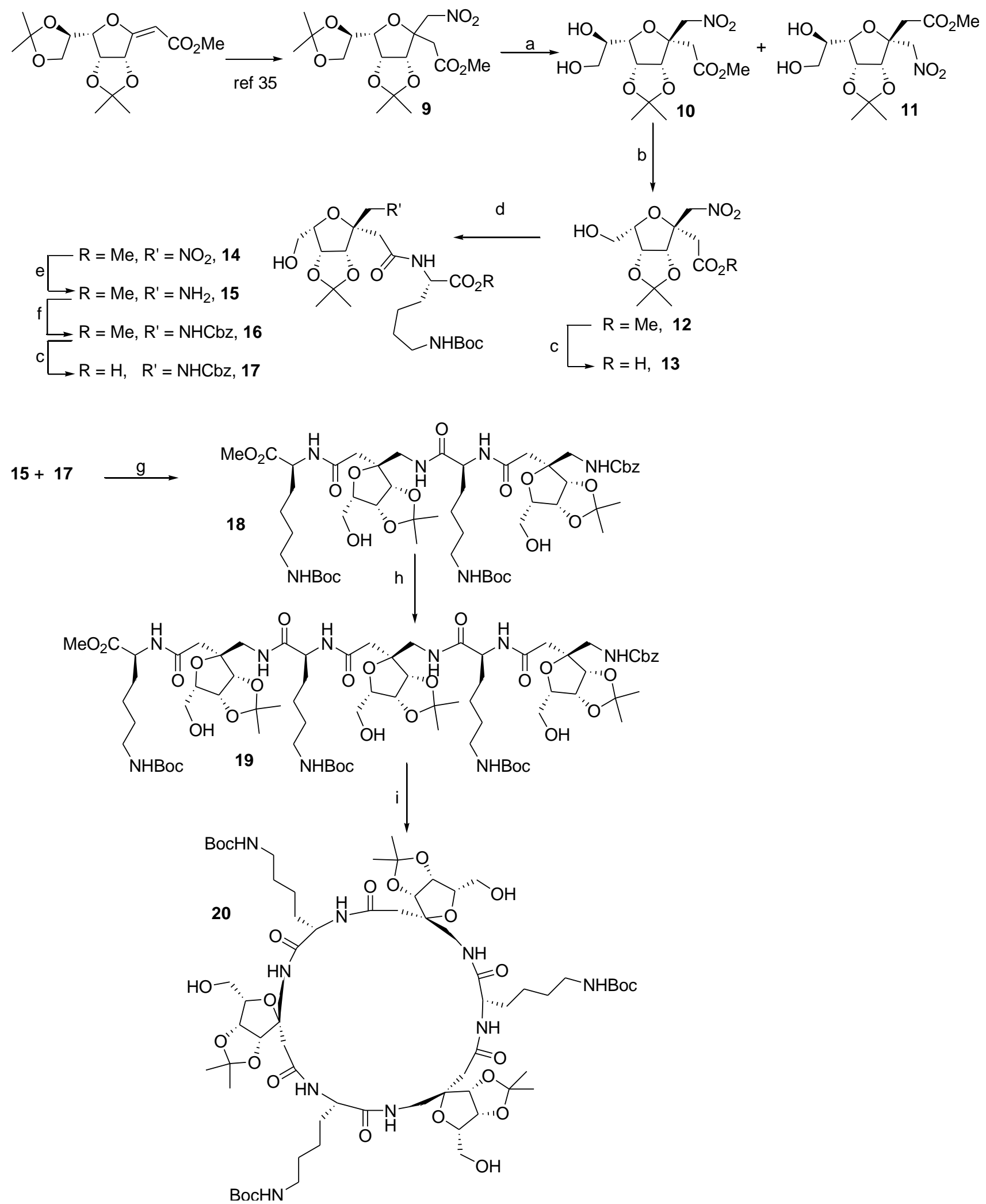

Scheme 2. a) $1 \mathrm{~N} \mathrm{HCl}, \mathrm{MeOH}, 0^{\circ} \mathrm{C}, 68 \%$; b) i. $\mathrm{NaIO}_{4}, \mathrm{MeOH}$; ii. $\mathrm{NaBH}_{4}, \mathrm{MeOH}, 0^{\circ} \mathrm{C}, 98 \%$; c) $\mathrm{LiOH}, \mathrm{THF} / \mathrm{H}_{2} \mathrm{O}, 13$ 98\%, 17 99\%; d) HCl.H-L-Lys(Boc)-OMe, DIEA, HATU, DMF, $0^{\circ} \mathrm{C}$, 90\%; e) $\mathrm{H}_{2}, \mathrm{Pd} / \mathrm{C}, \mathrm{MeOH}, 99 \%$; f) DIEA, CbzCl, DCM, $0^{\circ} \mathrm{C}, 70 \%$; g) HATU, DIEA, DMF, 
$0^{\circ} \mathrm{C}, 69 \%$; h) i. $\mathrm{H}_{2}, \mathrm{Pd} / \mathrm{C}, \mathrm{MeOH}$, ii. 17, HATU, DIEA, DMF, $0^{\circ} \mathrm{C}, 47 \%$; i) i. $\mathrm{LiOH}$, $\mathrm{THF} / \mathrm{H}_{2} \mathrm{O}$, ii. $\mathrm{H}_{2}, \mathrm{Pd} / \mathrm{C}, \mathrm{MeOH}$, , iii. HATU, DIEA, DMF/ $\mathrm{CH}_{2} \mathrm{Cl}_{2}, 0^{\circ} \mathrm{C}, 22 \%$.

To go further with these macrocyclic structures, we envisaged to modulate their hydrosolubility by removing the sugar hydroxyl protecting groups, namely 4,5isopropylidene. Moreover, these hydroxyls could also be considered as additional functionalization points. The cycloglycopeptide 8 was treated by a mixture of TFA/ $\mathrm{H}_{2} \mathrm{O}(1 / 1)$ at $0^{\circ} \mathrm{C}$, commonly used for isopropylidine removal (Scheme 3). Unexpectedly, we only observed the formation of $C$-glycosyl lactone $\mathbf{2 1}$ in quantitative yield, as a result of several concommitant intramolecular amide-to-ester conversions of $\mathbf{8}$. We postulated that compound 21 results from TFA promoted acetal removal followed by nucleophilic attack of O4 on carboxamide function. Few intramolecular lactonizations of $C$-glycosyl ester derivatives have already been described in the literature and led to the formation of $C$-glycosyl bicyclic lactones where the lactone ring is annulated to a furanoid ${ }^{42}$ or a pyranoide ${ }^{43}$ system. However, to the best of our knowledge, no lactonization of amide derivatives in such saccharidic systems has been described. This prompted us to study this reaction on less elaborate precursors in order to find experimental conditions enabling deprotection while preventing lactonization (Scheme 4). We first envisioned the 4,5-isopropylidene removal on $\beta$-nitro ester 10 (major isomer with methyl carboxylate and isopropylidene on the same face). As expected, the lactone 22 was obtained by treating 10 with a TFA $/ \mathrm{H}_{2} \mathrm{O}$ mixture at $0^{\circ} \mathrm{C}$, and no traces of nitro ester $\mathbf{2 3}$ were detected. The same reaction performed on $\alpha$-nitro ester $\mathbf{1 1}$ (minor anomer) gave the nitro ester $\mathbf{2 4}$ in quantitative yield without lactonization reaction since the methyl carboxylate and $\mathrm{O} 4$ are not on the same face. Taking into account the formation of lactone $\mathbf{2 1}$ resulting from intramolecular esterification of amide functions of $\mathbf{8}$, we planned to investigate the 4,5-isopropylidene removal on a simple amide like 25, obtained from $\mathbf{1 0}$ after saponification of methyl ester and coupling with H-L-ala-OMe (Scheme 4). To find efficient experimental conditions for 4,5-isopropylidene removal leading to compound $\mathbf{2 6}$ without concomitant lactonization, the nature of the acid, the solvent, the temperature and the reaction time were studied (Table 1). Treatment of 25 by a $1 / 1 \mathrm{TFA} / \mathrm{H}_{2} \mathrm{O}$ mixture led to $C$-glycosyl lactone 22 in quantitative yield (entry 1) although no reaction was observed while maintaining the temperature at $0^{\circ} \mathrm{C}$ (entry 2). Similarly, compound 26 was not formed when AcOH (entry 3), acidic resin (Amberlite IR 120, entry 4) ${ }^{44}$ or $\mathrm{FeCl}_{3}{ }^{45}$ (entry 5) were used. In these conditions, 30 to $50 \%$ of starting amide $\mathbf{2 5}$ were revovered and lactonization occurred up to $10 \%$. Moreover, prolonged reation time led to the degradation of the starting compound 25. 
We eventually investigated the use of $\mathrm{HCl}$ in $\mathrm{MeOH}$. When performed at $50^{\circ} \mathrm{C}$, the reaction only gave the lactone 22 in $82 \%$ yield in 2 hours (entry 6). TLC monitoring showed that maintaining the temperature at $-5^{\circ} \mathrm{C}$ could led to the expected 4,5-diol 26 without lactonization (compound $\mathbf{2 6}$ is more polar than lactone 22 on TLC) but this nitro ester was immediately converted into lactone 22 during evaporation of the solvent, even though the reaction mixture was neutralized with $\mathrm{NaHCO}_{3}$. Despite investigating various experimental conditions, it clearly appeared that lactonization of amide 25 during removal of the 4,5isopropylidene moiety could not be prevented. Consequently, removal of the isopropylidene group for hydrosolubility modulation or for additional functionalization was not an option in this context.

Table 1. 4,5-isopropylidene removal of $\mathbf{2 5}$

\begin{tabular}{|c|c|c|c|c|c|c|}
\hline entry & solvent & acid & temperature & $\begin{array}{l}\text { reaction } \\
\text { time }(h)\end{array}$ & $\begin{array}{l}\text { yield of } \\
22(\%)\end{array}$ & $\begin{array}{c}\text { recovery of } \\
25(\%)\end{array}$ \\
\hline 1 & $\mathrm{H}_{2} \mathrm{O}$ & TFA & $0^{\circ} \mathrm{C}$ then $\mathrm{rt}$ & 7 & 99 & 0 \\
\hline 2 & $\mathrm{H}_{2} \mathrm{O}$ & TFA & $0^{\circ} \mathrm{C}$ & 7 & 0 & 99 \\
\hline 3 & $\mathrm{H}_{2} \mathrm{O}$ & $\mathrm{AcOH}$ & $50^{\circ} \mathrm{C}$ & 48 & $<10$ & 50 \\
\hline 4 & $\mathrm{CHCl}_{3} / \mathrm{MeOH}$ & acidic resin & $40^{\circ} \mathrm{C}$ & 48 & $<10$ & 30 \\
\hline 5 & $\mathrm{CH}_{2} \mathrm{Cl}_{2}$ & $\mathrm{FeCl}_{3} \cdot \mathrm{H}_{2} \mathrm{O}$ & $40^{\circ} \mathrm{C}$ & 48 & $<10$ & 30 \\
\hline 6 & $\mathrm{MeOH}$ & $\mathrm{HCl}$ & $50^{\circ} \mathrm{C}$ & 2 & 82 & 0 \\
\hline 7 & $\mathrm{MeOH}$ & $\mathrm{HCl}$ & $0^{\circ} \mathrm{C}$ then rt & 7 & 80 & 20 \\
\hline 8 & $\mathrm{MeOH}$ & $\mathrm{HCl}$ & $-5^{\circ} \mathrm{C}$ & 12 & $80^{*}$ & 20 \\
\hline
\end{tabular}

"The reaction mixture was maintained at $-5^{\circ} \mathrm{C}$ during $12 \mathrm{~h}$ and then neutralized with $\mathrm{NaHCO}_{3}$. The formation of the lactone was observed during evaporation of the solvent.
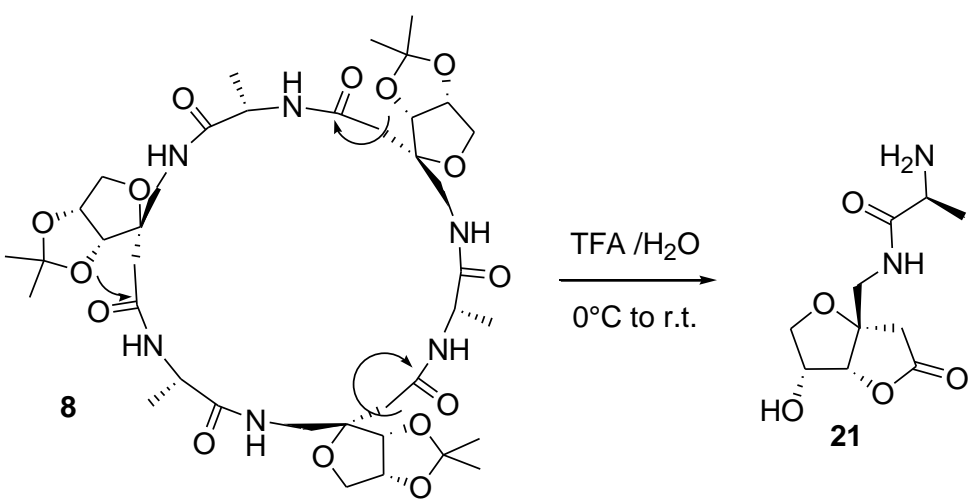

Scheme 3. Deprotection of cyclopeptide 8 

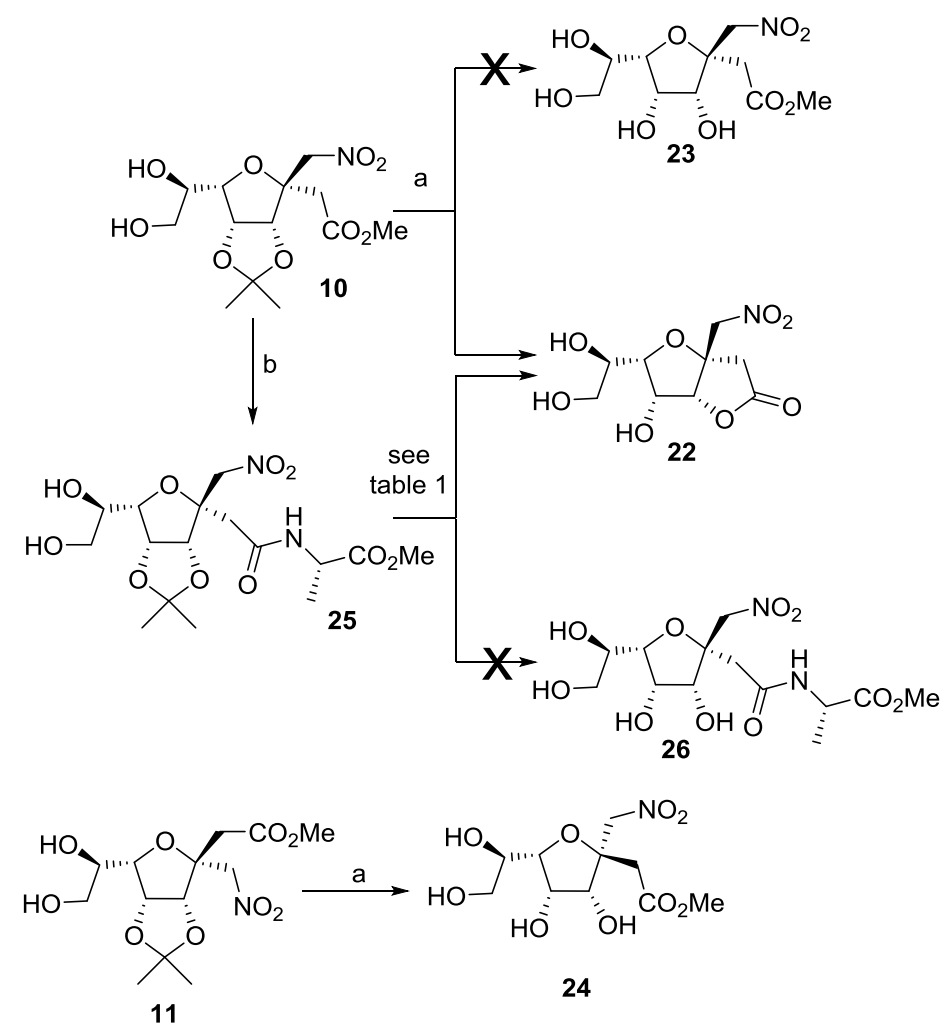

Scheme 4. a: TFA $/ \mathrm{H}_{2} \mathrm{O},(1 / 1), 0^{\circ} \mathrm{C}$; quantitative yield; b) i. $\mathrm{LiOH}, \mathrm{THF} / \mathrm{H}_{2} \mathrm{O}, 74 \%$, ii. $\mathrm{H}-\mathrm{L}-$ alaOMe, DIEA, HATU, DMF, $40 \%$; c) $\mathrm{HCl} / \mathrm{MeOH}, 0^{\circ} \mathrm{C}, 82 \%$.

These results unambiguously suggested that the minor $\alpha$-anomer 11 displaying the methyl carboxylate on one side of the sugar ring and the isopropylidene on the other side could be a valuable unit for cyclic hybrid glycopeptides synthesis and efficient isopropylidene deprotection. A similar pathway was thus investigated starting from 11 (see SI, scheme S1). However, it is important to note that compound $\mathbf{1 1}$ was obtained in 15\% yield after addition of nitromethane anion (minor anomer). Furthermore, after preliminary intramolecular coupling experiments, the cyclic product could not be isolated. The nature and structure of the linear precursor clearly affected the macrocyclization process. In particular, the anomeric configuration did not seem to induce a favorable folding of this linear precursor or provide the required spatial arrangement between amino and carboxylic acid moieties. Moreover, the steric hindrance around the anomeric aminomethyl moiety (on the same face of isopropylidene and hydroxymethyl chain) could prevent the cyclization reaction.

\section{Conclusion}


To sum up this work, we have synthesized new and original cyclic glycopeptides containing two types of amino acids, a $\gamma$-glycoamino acid and an $\alpha$-amino acid. The construction of the linear precursors based on two building blocks was achieved by a multistep sequence. A first macrocycle was obtained as a proof of concept to validate the cyclization capabilities of these glycopeptides. The second structure is more elaborate and has proved challenging to synthetize and purify. Nevertheless, this later presents an extended number of chemical functionalities, with the idea of exploring their scope as template for multivalency. During acid catalyzed removal of isopropylidene of these new cyclic glycopeptides, a $C$-glycosyl lactone in which the lactone ring is annulated to a furanoid system was unexpectedly and spontaneously formed. Intense investigations are now devoted to functionalization of these cyclopeptides via coupling of the amino or hydroxymethyl moieties to relevant biomolecules and/or dyes for biological applications.

\section{Experimental part}

\section{Reagents and general methods}

DMF was dried by distillation from calcium hydride. Other solvents and reagents were purchased from commercial sources and used without further purification. TLC analyses were performed using standard procedures on Kieselgel 60 F254 plates (Merck). Compounds were visualized using UV light $(254 \mathrm{~nm})$ and $30 \%$ methanolic $\mathrm{H}_{2} \mathrm{SO}_{4} /$ heat as developing agent. Column chromatography was performed on silica gel SI 60 (63-200 $\mu \mathrm{m})$ or Lichroprep RP-18 $(40-63 \mu \mathrm{m})($ Merck). FTIR spectra were recorded on a Perkin-Elmer spectrum 1000 on $\mathrm{NaCl}$ windows (film) or $\mathrm{KBr}$ pellets $\left(\mathrm{cm}^{-1}\right)$. Melting points were determined with a Tottoli apparatus and are uncorrected. Optical rotations were measured on an Anton Paar MC300 polarimeter. ${ }^{1} \mathrm{H}$ and ${ }^{13} \mathrm{C}$ NMR spectra were recorded on a Bruker spectrometer DPX250 (250 $\mathrm{MHz}$ and 62.9 MHz, respectively) and DRX400 (400 MHz and 100.6 MHz, respectively). For complete assignment of ${ }^{1} \mathrm{H}$ and ${ }^{13} \mathrm{C}$ signals, two-dimensional ${ }^{1} \mathrm{H},{ }^{1} \mathrm{H} \mathrm{COSY}$ and ${ }^{1} \mathrm{H},{ }^{13} \mathrm{C}$ correlation spectra were recorded. Mass spectra (MS) were recorded on an ESI/QqTOF Bruker spectrometer. Ethyl acetate $=\mathrm{EA}$, Cyclohexane $=$ cyH. Compounds 1, 2, 9, 10, 11 were prepared in accordance with literature procedures. ${ }^{35}$

\section{Procedure A: General procedure for peptidic coupling with HATU}


To a solution of amine (1.1 mmol, 1.1 eq.) and carboxylic acid (1 mmol, 1 eq.) in DMF (10 $\mathrm{mL}$ ) were added HATU (570 mg, $1.5 \mathrm{mmol}, 1.5$ eq.) and DIEA (363 $\mu \mathrm{L}, 2.1 \mathrm{mmol}, 2.1$ eq.) at $0^{\circ} \mathrm{C}$ under argon atmosphere. After stirring at room temperature for $24 \mathrm{~h}$, the solvent was evaporated. The residue was dissolved in ethyl acetate $(20 \mathrm{~mL})$, washed with $\mathrm{HCl} 1 \mathrm{~N}(5 \mathrm{~mL})$, a saturated aqueous solution of $\mathrm{NaHCO}_{3}(5 \mathrm{~mL})$ and brine $(5 \mathrm{~mL})$. The organic layer was dried over $\mathrm{MgSO}_{4}$, filtered and concentrated. The product was purified by column chromatography (silica gel, cyH/EA).

\section{Procedure B: General procedure for saponification with $\mathrm{LiOH}$}

To a solution of methyl ester $(2 \mathrm{mmol})$ in THF $(15 \mathrm{~mL})$ and water $(5 \mathrm{~mL})$ was added $\mathrm{LiOH}$ (144 mg, $6 \mathrm{mmol}, 3$ eq.) and the mixture was stirred until completion of the reaction. THF was removed under reduced pressure, ethyl acetate $(20 \mathrm{~mL})$ was added and the $\mathrm{pH}$ was adjusted to 2-3 with aqueous $1 \mathrm{~N} \mathrm{HCl}$. The organic layer was washed with brine and dried over $\mathrm{MgSO}_{4}$, filtered and concentrated. When necessary, the crude residue was purified by column chromatography (silica gel, cyH/EA).

\section{Procedure C: General procedure for nitro reduction or Cbz hydrogenolysis}

To a solution of protected compound $(10 \mathrm{mmol})$ in $\mathrm{MeOH}(20 \mathrm{~mL})$ was added $\mathrm{Pd} / \mathrm{C}(10 \%)$ $(40 \% \mathrm{w} / \mathrm{w})$. The mixture was hydrogenated under $\mathrm{H}_{2}$ atmosphere (40 psi) for $24 \mathrm{~h}$. The reaction mixture was then filtered through a pad of celite and concentrated to give the desired product. The crude product was used without further purification.

\section{Compound 3}

The above described procedure A was applied to $2(580 \mathrm{mg}, 1.59 \mathrm{mmol})$ and alanine methyl ester hydrochloride to provide $3(608 \mathrm{mg}, 85 \%$ yield $)$. Colorless gum. $\mathrm{R} f=0.58(\mathrm{EA}),[\alpha]_{\mathrm{D}}=-$ 30.9 (c 0.5 in $\left.\mathrm{CHCl}_{3}\right) .{ }^{1} \mathrm{H} \mathrm{NMR}\left(\mathrm{CDCl}_{3}, 250 \mathrm{MHz}\right): \delta=1.33$ (s, 3H, $\left.\mathrm{CH}_{3}\right), 1.38(\mathrm{~d}, 3 \mathrm{H}, J=$ $7.0 \mathrm{~Hz}, \mathrm{CH}_{3}$ alanine), $1.52\left(\mathrm{~s}, 3 \mathrm{H}, \mathrm{CH}_{3}\right), 2.61\left(\mathrm{~d}, 1 \mathrm{H}, J_{\mathrm{gem}}=14.0 \mathrm{~Hz}, H-2\right), 2.67$ (d, $\left.1 \mathrm{H}, H-2\right)$, $3.20\left(\mathrm{dd}, 1 \mathrm{H}, J_{\mathrm{gem}}=14.5, J_{2}{ }^{\prime}, \mathrm{NH}=5.5 \mathrm{~Hz}, H-2^{\prime}\right), 3.37\left(\mathrm{dd}, 1 \mathrm{H}, J_{2}{ }^{\prime}, \mathrm{NH}=7.5 \mathrm{~Hz}, H-2^{\prime}\right), 3.72(\mathrm{~s}$, $3 \mathrm{H}, \mathrm{OMe}), 3.92\left(\mathrm{dd}, 1 \mathrm{H}, J_{\mathrm{gem}}=11.0, J_{5,6}=3.5 \mathrm{~Hz}, H-6\right), 4.01(\mathrm{~d}, 1 \mathrm{H}, H-6), 4.46-4.57$ (m, 2H, $H-\alpha, H-4), 4.90\left(\mathrm{dd}, 1 \mathrm{H}, J_{4,5}=6.0 \mathrm{~Hz}, H-5\right), 5.06\left(\mathrm{~d}, 1 \mathrm{H}, J_{\mathrm{gem}}=12.0 \mathrm{~Hz}, \mathrm{CH}_{2}-\mathrm{Ph}\right), 5.12(\mathrm{~d}$, $\left.1 \mathrm{H}, \mathrm{CH}_{2}-\mathrm{Ph}\right), 5.66(\mathrm{t}, 1 \mathrm{H}, \mathrm{N}-H), 6.80\left(\mathrm{~d}, 1 \mathrm{H}, J_{\alpha, \mathrm{NH}}=6.5 \mathrm{~Hz}, \mathrm{~N}-H\right), 7.29-7.40(\mathrm{~m}, 5 \mathrm{H}, H-\mathrm{Ar})$. ${ }^{13} \mathrm{C} \mathrm{NMR}\left(\mathrm{CDCl}_{3}, 62.9 \mathrm{MHz}\right): \delta=17.9\left(\mathrm{CH}_{3}\right.$ alanine $\left.), 24.6,26.1\left(2 \mathrm{C}\left(\mathrm{CH}_{3}\right)_{2}\right)\right), 40.3(C-2)$, 42.1 (C-2'), 48.1 (C- $\alpha), 52.4\left(\mathrm{OCH}_{3}\right), 66.8\left(\mathrm{CH}_{2}-\mathrm{Ph}\right), 71.4(C-6), 81.4,83.0(C-4, C-5), 85.6$ $\left(C\right.$-3), $112.6\left(C\left(\mathrm{CH}_{3}\right)_{2}\right), 128.0$ (2 $C$-Ar), 128.5 (3 $C$-Ar), $136.4(C$-Ar), $156.8(C=\mathrm{O} \mathrm{Cbz})$, 
$170.4(C=\mathrm{O}), 173.2(C=\mathrm{O})$. ESI-HRMS $[\mathrm{M}+\mathrm{Na}]^{+} \mathrm{m} / \mathrm{z}=$ found 473.1902 (calculated for $\mathrm{C}_{22} \mathrm{H}_{30} \mathrm{~N}_{2} \mathrm{O}_{8} \mathrm{Na}$ : 473.1894). IR (NaCl) $\mathrm{v}_{\max } / \mathrm{cm}^{-1} 3341,1725,1662$

\section{Compound 6}

The above described procedure B was applied to $\mathbf{3}$ and acid $\mathbf{4}$ was obtained in quantitative yield. The amine 5 was obtained from 3 in $99 \%$ yield via procedure C. Acid 4 (392 mg, 0.9 mmol, 1 eq.) and amine 5 (313 mg, 0.99 mmol, 1.1 eq.) were coupled according to procedure A to provide 6 (411 mg, 78\% yield). Colorless gum. $\mathrm{R} f=0.29(\mathrm{EA}) .[\alpha]_{\mathrm{D}}=-33.3^{\circ}$ (c 1 in $\left.\mathrm{CHCl}_{3}\right) .{ }^{1} \mathrm{H} \mathrm{NMR}\left(\mathrm{CDCl}_{3}, 400 \mathrm{MHz}\right): \delta=1.32,1.34\left(\mathrm{~s}, 6 \mathrm{H}, \mathrm{C}\left(\mathrm{CH}_{3}\right)_{2}\right), 1.37$ (d, 6H, $J=7.0$ $\mathrm{Hz}, \mathrm{CH}_{3}$ alanine), 1.49, $1.52\left(\mathrm{~s}, 6 \mathrm{H}, \mathrm{C}\left(\mathrm{CH}_{3}\right)_{2}\right), 2.64\left(\mathrm{~s}, 2 \mathrm{H}, H_{\mathrm{A}}-2\right), 2.65\left(\mathrm{~d}, 1 \mathrm{H}, J_{\text {gem }}=14.5 \mathrm{~Hz}\right.$, $\left.H_{\mathrm{B}}-2\right), 2.70\left(\mathrm{~d}, 1 \mathrm{H}, H_{\mathrm{B}}-2\right), 3.19\left(\mathrm{dd}, 1 \mathrm{H}, J_{\text {gem }}=14.5, J=6.0 \mathrm{~Hz}, H_{\mathrm{B}}-2^{\prime}\right), 3.26\left(\mathrm{dd}, 1 \mathrm{H}, J_{\text {gem }}=\right.$ $\left.14.5, J=6.0 \mathrm{~Hz}, H_{\mathrm{A}}-2^{\prime}\right), 3.38\left(\mathrm{dd}, 1 \mathrm{H}, J=7.0 \mathrm{~Hz}, H_{\mathrm{B}}-2^{\prime}\right), 3.42\left(\mathrm{dd}, 1 \mathrm{H}, J=7.0 \mathrm{~Hz}, H_{\mathrm{A}}-2^{\prime}\right)$, 3.74 (s, 3H, OCH$\left.H_{3}\right), 3.90-4.01$ (m, 4H, H-6), 4.41-4.54 (m, 3H, $\left.2 H-\alpha, H-4\right), 4.57$ (d, 1H, $J_{4,5}=$ $6.0 \mathrm{~Hz}, H-4), 4.88-4.91$ (m, 2H, H-5), 5.10 (s, 2H, CH2-Ph), 6.03 (t, $1 \mathrm{H}, J=6.5 \mathrm{~Hz}, \mathrm{NHCbz}$ ), $6.71\left(\mathrm{~d}, 1 \mathrm{H}, J=7.0 \mathrm{~Hz}, \mathrm{~N}_{3} H\right), 6.86\left(\mathrm{~d}, 1 \mathrm{H}, J=7.0 \mathrm{~Hz}, \mathrm{~N}_{1} H\right), 7.19\left(\mathrm{t}, 1 \mathrm{H}, \mathrm{N}_{2} H\right), 7.27-7.37$ (m, $5 \mathrm{H}, H$-Ar). ${ }^{13} \mathrm{C}$ NMR $\left(\mathrm{CDCl}_{3}, 100 \mathrm{MHz}\right): \delta=17.6,17.7$ (2 $\mathrm{CH}_{3}$ alanine), 24.6, $26.1\left(4 \mathrm{CH}_{3}\right)$, $39.5\left(C_{\mathrm{B}}-2\right), 39.9\left(C_{\mathrm{A}}-2\right), 40.2\left(C_{\mathrm{A}}-2\right.$ '), $42.2\left(C_{\mathrm{B}}-2\right.$ '), $48.2(\mathrm{C}-\alpha), 49.2(\mathrm{C}-\alpha), 52.5\left(\mathrm{OCH}_{3}\right)$, $66.8\left(\mathrm{CH}_{2}-\mathrm{Ph}\right), 71.4,71.5\left(C_{\mathrm{A}}-6, C_{\mathrm{B}}-6\right), 81.3,81.4\left(C_{\mathrm{B}}-5, C_{\mathrm{A}}-5\right), 83.1,83.4\left(C_{\mathrm{B}}-4, C_{\mathrm{A}}-4\right)$, 85.5, $85.8\left(C_{\mathrm{B}}-3, C_{\mathrm{A}}-3\right), 112.5\left(2 C\left(\mathrm{CH}_{3}\right)_{2}\right), 128.0$ (3 $C$-Ar), 128.5 (2 $C$-Ar), $136.5(C$-Ar), $157.1(C=\mathrm{O} \mathrm{Cbz}), 170.6\left(C_{4}=\mathrm{O}\right), 170.8\left(C_{2}=\mathrm{O}\right), 172.6\left(C_{3}=\mathrm{O}\right), 173.5\left(C_{1}=\mathrm{O}\right)$. ESI-HRMS $[\mathrm{M}+\mathrm{Na}]^{+} \mathrm{m} / \mathrm{z}=$ found 757.3257 (calculated for $\mathrm{C}_{35} \mathrm{H}_{50} \mathrm{~N}_{4} \mathrm{O}_{13} \mathrm{Na}:$ 757.3267). IR $(\mathrm{NaCl}) \mathrm{u}_{\max } / \mathrm{cm}^{-1} 3322,2985,2938,1721,1659,1532$

\section{Compound 7}

Compound 6 was deprotected using general procedure $\mathrm{C}$ and the corresponding amine (396 $\mathrm{mg}, 0.66 \mathrm{mmol}, 1.1$ eq.) was coupled with acid 4 (262 mg, $0.6 \mathrm{mmol}, 1$ eq.) following procedure A to provide 7 (482 $\mathrm{mg}, 79 \%$ yield). Colorless gum $\mathrm{R} f=0.17(\mathrm{EA})$. $[\alpha]_{\mathrm{D}}=-35.2^{\circ}$ (c 0.6 in $\left.\mathrm{CHCl}_{3}\right) .{ }^{1} \mathrm{H}$ NMR $\left(\mathrm{CDCl}_{3}, 400 \mathrm{MHz}\right): \delta=1.30-1.35\left(\mathrm{~m}, 12 \mathrm{H}, 4 \mathrm{CH}_{3}\right), 1.37$ (d, 3H, $J$ $=7.5 \mathrm{~Hz}, \mathrm{CH}_{3}$ alanine $), 1.42\left(\mathrm{~d}, 3 \mathrm{H}, J=7.5 \mathrm{~Hz}, \mathrm{CH}_{3}\right.$ alanine $), 1.48\left(\mathrm{~s}, 3 \mathrm{H}, \mathrm{CH}_{3}\right), 1.51(\mathrm{~s}, 6 \mathrm{H}$, $\left.2 \mathrm{CH}_{3}\right), 2.59-2.75(\mathrm{~m}, 6 \mathrm{H}, 6 \mathrm{H}-2), 3.12\left(\mathrm{dd}, 1 \mathrm{H}, J_{\text {gem }}=14.5, J=5.0 \mathrm{~Hz}, H-2\right.$ ') 3.23 (dd, $1 \mathrm{H}$, $\left.J_{\text {gem }}=14.5, J=6.0 \mathrm{~Hz}, H-2^{\prime}\right), 3.30\left(\mathrm{dd}, 1 \mathrm{H}, J_{\text {gem }}=14.5, J=5.5 \mathrm{~Hz}, H-2^{\prime}\right), 3.39$ (dd, $1 \mathrm{H}, J=$ $\left.6.5 \mathrm{~Hz}, H-2^{\prime}\right), 3.43$ (dd, $1 \mathrm{H}, J=6.5 \mathrm{~Hz}, H-2$ ') 3.51 (dd, $1 \mathrm{H}, J=7.0 \mathrm{~Hz}, H-2$ ') 3.75 (s, 3H, $\left.\mathrm{OCH}_{3}\right), 3.90\left(\mathrm{dd}, 1 \mathrm{H}, J_{\mathrm{gem}}=11.0, J=4.0 \mathrm{~Hz}, H-6\right), 3.93-3.99(\mathrm{~m}, 5 \mathrm{H}, 5 H-6), 4.39(\mathrm{~m}, 1 \mathrm{H}, H-$ a), 4.44-4.55 (m, 4H, $2 H-\alpha, 2 H-4), 4.57$ (d, 1H, $\left.J_{4,5}=6.0 \mathrm{~Hz}, H-4\right), 4.87-4.92$ (m, 3H, $3 H-$ 
5), $5.08\left(\mathrm{~d}, 1 \mathrm{H}, J_{\text {gem }}=12.5 \mathrm{~Hz}, \mathrm{CH}_{2}-\mathrm{Ph}\right), 5.12\left(\mathrm{~d}, 1 \mathrm{H}, \mathrm{CH}_{2}-\mathrm{Ph}\right), 6.06(\mathrm{t}, 1 \mathrm{H}, J=6.0 \mathrm{~Hz}$, $\mathrm{NHCbz}), 6.74(\mathrm{~d}, 1 \mathrm{H}, J=7.0 \mathrm{~Hz}, \mathrm{NH}), 6.88(\mathrm{~d}, 1 \mathrm{H}, J=7.0 \mathrm{~Hz}, \mathrm{NH}), 7.07$ (d, 1H, J=7.0 Hz, $\mathrm{NH}), 7.25(\mathrm{~m}, 1 \mathrm{H}, \mathrm{NH}), 7.30-7.39(\mathrm{~m}, 5 \mathrm{H}, 5 \mathrm{H}-\mathrm{Ar}), 7.46(\mathrm{t}, 1 \mathrm{H}, J=6.0 \mathrm{~Hz}, \mathrm{NH}) .{ }^{13} \mathrm{C} \mathrm{NMR}$ $\left(\mathrm{CDCl}_{3}, 100 \mathrm{MHz}\right): \delta=17.5,17.7,18.0\left(3 \mathrm{CH}_{3}\right.$ alanine $), 24.6\left(3 \mathrm{CH}_{3}\right), 26.1\left(3 \mathrm{CH}_{3}\right), 39.2$, 39.7, 39.8, 40.1, 40.7, 41.9 (3 C-2, 3 C-2'), 48.2, 49.2, 49.4 (3 C- $\alpha$ ), $52.5\left(\mathrm{OCH}_{3}\right), 66.8\left(\mathrm{CH}_{2^{-}}\right.$ Ph), 71.2, 71.4, 71.6 (3 C-6), 81.2, 81.3, 81.4 (3 C-5), 83.1, 83.3, 83.4 (3 C-4), 85.5, 85.7, 85.8 (3 C-3), 112.4, 112.5 (3 $\left.C\left(\mathrm{CH}_{3}\right)_{2}\right), 128.0$ (3 $C$-Ar), 128.5 (2 $C$-Ar), 136.6 (C-Ar), 157.1 $(C=\mathrm{O} \mathrm{Cbz}), 170.5,170.7,171.2,172.9,173.5(6 C=\mathrm{O})$. ESI-HRMS $[\mathrm{M}+\mathrm{Na}]^{+} \mathrm{m} / \mathrm{z}=$ found 1041.4649 (calculated for $\left.\mathrm{C}_{48} \mathrm{H}_{70} \mathrm{~N}_{6} \mathrm{O}_{18} \mathrm{Na}: 1041.4639\right)$. IR $(\mathrm{NaCl}) \mathrm{U}_{\max } / \mathrm{cm}^{-1} 3327,2985$, $2938,1718,1657,1529$

\section{Cyclopeptide 8}

Compound 7 was deprotected using general procedures $\mathrm{B}$ and C. To a solution of the deprotected glyco-amino acid (348 mg, $0.4 \mathrm{mmol}, 1$ eq.) in $\mathrm{CH}_{2} \mathrm{Cl}_{2} / \mathrm{DMF}$ (4/1) at $0^{\circ} \mathrm{C}$ under argon, were added DIEA (346 $\mu \mathrm{L}, 2.0 \mathrm{mmol}, 5.0$ eq.) and HATU (182 mg, $0.48 \mathrm{mmol}, 1.2$ eq.). After stirring at room temperature for 3 days, the solvents were evaporated and the residue was taken up in ethyl acetate $(30 \mathrm{~mL})$. The solution was washed with brine $(10 \mathrm{~mL})$, dried over $\mathrm{MgSO}_{4}$, filtered and concentrated. The product was purified by column chromatography (silica gel, AcOEt/MeOH) to provide 8 (143 mg, 42\% yield). White solid. $\mathrm{Mp}=180{ }^{\circ} \mathrm{C} . \mathrm{R} f=0.33\left(\mathrm{CH}_{2} \mathrm{Cl}_{2} / \mathrm{MeOH}: 9 / 1\right) .[\alpha]_{\mathrm{D}}=-50.5^{\circ}\left(\mathrm{c} 0.5\right.$ in $\left.\mathrm{CHCl}_{3}\right) .{ }^{1} \mathrm{H} \mathrm{NMR}$ $\left(\mathrm{CDCl}_{3}, 250 \mathrm{MHz}\right): \delta=1.36\left(\mathrm{~s}, 3 \mathrm{H}, \mathrm{CH}_{3}\right), 1.42\left(\mathrm{~d}, 3 \mathrm{H}, J=7.0 \mathrm{~Hz}, \mathrm{CH}_{3}\right.$ alanine $), 1.53(\mathrm{~s}, 3 \mathrm{H}$, $\left.\mathrm{CH}_{3}\right), 2.64\left(\mathrm{~d}, 1 \mathrm{H}, J_{\text {gem }}=14.5 \mathrm{~Hz}, H-2\right), 2.79(\mathrm{~d}, 1 \mathrm{H}, H-2), 3.32\left(\mathrm{dd}, 1 \mathrm{H}, J_{\text {gem }}=14.5, J_{2}{ }^{\prime}, \mathrm{NH}=\right.$ $\left.6.0 \mathrm{~Hz}, H-2^{\prime}\right), 3.47\left(\mathrm{dd}, 1 \mathrm{H}, J_{2}{ }^{\prime}, \mathrm{NH}=6.0 \mathrm{~Hz}, H-2^{\prime}\right), 3.98\left(\mathrm{~d}, 1 \mathrm{H}, J_{\text {gem }}=11.5 \mathrm{~Hz}, H-6\right), 4.05$ (dd, $\left.1 \mathrm{H}, J_{5,6}=3.5 \mathrm{~Hz}, H-6^{\prime}\right), 4.48(\mathrm{~m}, 1 \mathrm{H}, H-\alpha), 4.51$ (d, $\left.1 \mathrm{H}, J_{4,5}=6.0 \mathrm{~Hz}, H-4\right), 4.94$ (dd, $1 \mathrm{H}$, $H-5), 6.72\left(\mathrm{~d}, 1 \mathrm{H}, J=6.0 \mathrm{~Hz}, \mathrm{NH}\right.$ ala), 7.40 (t, $1 \mathrm{H}, \mathrm{NH}$ erythro). ${ }^{13} \mathrm{C} \mathrm{NMR}\left(\mathrm{CDCl}_{3}, 62.9\right.$ $\mathrm{MHz}): \delta=17.8\left(\mathrm{CH}_{3}\right.$ alanine $), 24.8\left(\mathrm{CH}_{3}\right), 26.3\left(\mathrm{CH}_{3}\right), 40.7,41.0\left(C-2, C-2{ }^{\prime}\right), 49.6(\mathrm{C}-\alpha)$, $71.7(C-6), 81.6(C-5), 83.4(C-4), 85.9(C-3), 112.8\left(C\left(\mathrm{CH}_{3}\right)_{2}\right), 171.5,173.2(2 C=\mathrm{O})$. ESIHRMS $[\mathrm{M}+\mathrm{Na}]^{+} \mathrm{m} / \mathrm{z}=$ found 875.4012 (calculated for $\mathrm{C}_{39} \mathrm{H}_{60} \mathrm{~N}_{6} \mathrm{O}_{15} \mathrm{Na}:$ 875.4009). IR $(\mathrm{NaCl}) v_{\max } / \mathrm{cm}^{-1} 3312,3056,2981,2933,2857,1737,1657,1529$

\section{Compound 12}

To a stirred solution of $\mathbf{1 0}\left(5.42 \mathrm{~g}, 16.2 \mathrm{mmol}, 1\right.$ eq.) in methanol (340 mL) was added $\mathrm{NaIO}_{4}$ ( $6.93 \mathrm{~g}, 32.4 \mathrm{mmol}, 2$ eq.) under argon. After stirring at room temperature until completion of the reaction, the solvent was removed by half in vacuo. The mixture was diluted with $\mathrm{CH}_{2} \mathrm{Cl}_{2}$ 
(300 mL). The organic layer was washed with water $(3 \times 100 \mathrm{~mL})$, dried over $\mathrm{MgSO}_{4}$, filtered and the solvent was removed under reduced pressure. The crude product was solubilized in $\mathrm{MeOH}(130 \mathrm{~mL})$ and $\mathrm{NaBH}_{4}(622 \mathrm{mg}, 1.51 \mathrm{mmol}, 1$ eq. $)$ was added at $0^{\circ} \mathrm{C}$. After $1 \mathrm{~h}$ at room temperature, the solvent was removed under reduced pressure. The residue was diluted in $\mathrm{CH}_{2} \mathrm{Cl}_{2}(200 \mathrm{~mL})$ and the organic layer was washed with a solution of $1 \mathrm{~N} \mathrm{HCl}(70 \mathrm{~mL})$ and with water until $\mathrm{pH}=7$. The organic layer was dried over $\mathrm{MgSO}_{4}$, filtrered and evaporated under reduced pressure. The crude product 12 (5.10 g, 98\% yield) was used without further purification. Colorless oil. $\mathrm{R} f=0.50(\mathrm{cH} / \mathrm{EA}: 1 / 2) .[\alpha]_{\mathrm{D}}=-10.4^{\circ}\left(\mathrm{c} 0.4\right.$ in $\left.\mathrm{CHCl}_{3}\right) .{ }^{1} \mathrm{H} \mathrm{NMR}$ $\left(\mathrm{CDCl}_{3}, 250 \mathrm{MHz}\right): \delta=1.31\left(\mathrm{~s}, 3 \mathrm{H}, \mathrm{CH}_{3}\right), 1.48$ (s, 3H, $\left.\mathrm{CH}_{3}\right), 2.09$ (br s, $\left.1 \mathrm{H}, \mathrm{OH}\right), 2.90$ (d, $\left.1 \mathrm{H}, J_{\text {gem }}=18.0 \mathrm{~Hz}, H-2\right), 3.09(\mathrm{~d}, 1 \mathrm{H}, H-2), 3.73\left(\mathrm{~s}, 3 \mathrm{H}, \mathrm{OCH}_{3}\right), 3.83-3.90(\mathrm{~m}, 2 \mathrm{H}, H-7, H-$ 7'), 4.11 (m, H, H-6), 4.59 (d, 1H, $J_{\text {gem }}=11.5 \mathrm{~Hz}, H-2$ '), 4.67 (d, 1H, $\left.J_{4,5}=6.0 \mathrm{~Hz}, H-4\right), 4.90$ $\left(\mathrm{dd}, 1 \mathrm{H}, J_{5,6}=4.0 \mathrm{~Hz}, H-5\right), 5.06\left(\mathrm{~d}, 1 \mathrm{H}, H-2{ }^{\prime}\right) .{ }^{13} \mathrm{C} \mathrm{NMR}\left(\mathrm{CDCl}_{3}, 62.9 \mathrm{MHz}\right): \delta=24.5$ $\left(\mathrm{CH}_{3}\right), 25.8\left(\mathrm{CH}_{3}\right), 36.3(C-2), 52.0\left(\mathrm{OCH}_{3}\right), 61.0(C-7), 76.0(C-2), 79.7,81.4,83.7(C-4, C-$ 5, $C-6), 83.3(C-3), 113.6\left(C\left(\mathrm{CH}_{3}\right)_{2}\right), 171.1(C=\mathrm{O})$. ESI-HRMS $[\mathrm{M}+\mathrm{Na}]^{+} \mathrm{m} / \mathrm{z}=$ found 328.1014 (calculated for $\mathrm{C}_{12} \mathrm{H}_{19} \mathrm{NO}_{8} \mathrm{Na}$ : 328.1003). IR (NaCl) $U_{\max } / \mathrm{cm}^{-1} 3507,2985,2943$, 1735,1553

\section{Compound 14}

Compound 12 (5.10 g, $15.9 \mathrm{mmol}$ ) was saponified by $\mathrm{LiOH}$ (general procedure B) to provide 13 (4.78 g, 98\% yield). Compound 13 (4.78 g, 15.6 mmol) was coupled with H-L-Lys(Boc)OMe following procedure A to provide 14 (7.72 g, $90 \%)$. Colorless oil. R $f=0.21$ (cH/EA: 3/7). $[\alpha]_{\mathrm{D}}=-22.0^{\circ}$ (c 0.4 in $\left.\mathrm{CHCl}_{3}\right) .{ }^{1} \mathrm{H}$ NMR $\left(\mathrm{CDCl}_{3}, 250 \mathrm{MHz}\right): \delta=1.25-1.54(\mathrm{~m}, 4 \mathrm{H}, 2$ $\mathrm{CH}_{2}$ lys), 1.32 (s, 3H, $\left.\mathrm{CH}_{3}\right), 1.43$ (s, 9H, $\mathrm{C}\left(\mathrm{CH}_{3}\right)_{3}$ boc), 1.51 (s, 3H, $\left.\mathrm{CH}_{3}\right), 1.66-1.90$ (m, 2H, $\mathrm{CH}_{2}$ lys), 2.77 (d, $\left.1 \mathrm{H}, J_{\text {gem }}=16.0 \mathrm{~Hz}, H-2\right), 2.99-3.12$ (m, 3H, H-2, $\mathrm{CH}_{2}$ lys), 3.75 (s, 3H,

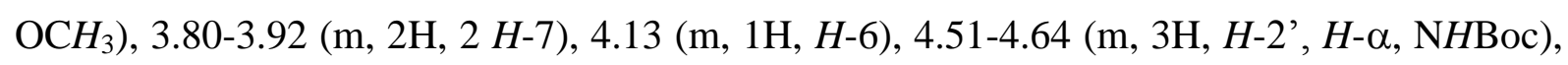
$4.79\left(\mathrm{~d}, 1 \mathrm{H}, J_{4,5}=6.0 \mathrm{~Hz}, H-4\right), 4.92\left(\mathrm{dd}, 1 \mathrm{H}, J_{5,6}=4.0 \mathrm{~Hz}, H-5\right), 5.09\left(\mathrm{~d}, 1 \mathrm{H}, J_{\text {gem }}=12.0 \mathrm{~Hz}\right.$, $\left.H-2^{\prime}\right), 6.49(\mathrm{~d}, 1 \mathrm{H}, J=8.0 \mathrm{~Hz}, \mathrm{NH}) .{ }^{13} \mathrm{C} \mathrm{NMR}\left(\mathrm{CDCl}_{3}, 62.9 \mathrm{MHz}\right): \delta=22.4\left(\mathrm{CH}_{2}\right.$ lys $), 24.3$ $\left(\mathrm{CH}_{3}\right), 25.8\left(\mathrm{CH}_{3}\right), 28.4\left(\mathrm{C}\left(\mathrm{CH}_{3}\right)_{3}\right.$ boc $), 29.5\left(\mathrm{CH}_{2}\right.$ lys $), 31.9\left(\mathrm{CH}_{2}\right.$ lys $), 38.5(C-2), 40.4\left(\mathrm{CH}_{2}\right.$ lys), 52.0, $52.5\left(C-\alpha, \mathrm{OCH}_{3}\right), 61.2(C-7), 76.7\left(C-2^{\prime}\right), 79.9,81.5\left(C\left(\mathrm{CH}_{3}\right)_{3}\right.$ boc, $\left.C-5, C-6\right), 83.4$ (C-3), $83.9(C-4), 113.3\left(C\left(\mathrm{CH}_{3}\right)_{2}\right), 156.2(C=\mathrm{O}$ boc), $169.4(C=\mathrm{O}), 172.7$ ( $C=\mathrm{O}$ lys). ESIHRMS $[\mathrm{M}+\mathrm{Na}]^{+} \mathrm{m} / \mathrm{z}=$ found 556.2485 (calculated for $\mathrm{C}_{23} \mathrm{H}_{39} \mathrm{~N}_{3} \mathrm{O}_{11} \mathrm{Na}$ : 556.2477). IR $(\mathrm{NaCl}) \cup_{\max } / \mathrm{cm}^{-1} 3364,2981,2933,2867,1742,1690,1666,1551,1529$

\section{Compound 16}


Compound 14 (3.85 g, $7 \mathrm{mmol}$ ) was deprotected according to general procedure $\mathrm{C}$ to provide amine 15 (3.52 g, $7 \mathrm{mmol}$, 99\%) which was then was then protected with a benzyloxycarbonyl group. The crude was suspended in $\mathrm{CH}_{2} \mathrm{Cl}_{2}(30 \mathrm{~mL})$ and DIEA $(2.18 \mathrm{~mL}$, $12.6 \mathrm{mmol}, 1.8$ eq.) followed by $\mathrm{Cbz}-\mathrm{Cl}(1.5 \mathrm{~mL}, 10.5 \mathrm{mmol}, 1.5$ eq. $)$ were added at $0^{\circ} \mathrm{C}$. The reaction mixture was allowed to warm to room temperature and stirred overnight. Solvent was removed under vacuum and the residue was dissolved in ethyl acetate $(40 \mathrm{~mL})$, washed with $0.5 \mathrm{M} \mathrm{HCl}(20 \mathrm{~mL})$ and brine $(20 \mathrm{~mL})$. The organic layer was dried over $\mathrm{MgSO}_{4}$, filtered and concentrated. The residue was purified by column chromatography (silica gel, cyH/EA) to provide $16(3.12 \mathrm{~g}, 70 \%)$. Colorless oil. $\mathrm{R} f=0.08(\mathrm{cH} / \mathrm{EA}: 1 / 1) .[\alpha]_{\mathrm{D}}=-1.8^{\circ}\left(\mathrm{c} 0.5\right.$ in $\left.\mathrm{CHCl}_{3}\right)$. ${ }^{1} \mathrm{H} \mathrm{NMR}\left(\mathrm{CDCl}_{3}, 250 \mathrm{MHz}\right): \delta=1.28-1.58$ (m, 4H, $\left.2 \mathrm{CH}_{2} \mathrm{lys}\right), 1.34$ (s, 3H, $\left.\mathrm{CH}_{3}\right), 1.47$ (s, 9H, $\mathrm{C}\left(\mathrm{CH}_{3}\right)_{3}$ boc), 1.54 (s, 3H, $\mathrm{CH}_{3}$ ), 1.66-1.92 (m, 2H, $\mathrm{CH}_{2}$ lys), 2.38 (br s, $1 \mathrm{H}, \mathrm{OH}$ ), 2.63 $\left(\mathrm{d}, 1 \mathrm{H}, J_{\text {gem }}=14.0 \mathrm{~Hz}, H-2\right), 2.72(\mathrm{~d}, 1 \mathrm{H}, H-2), 3.08-3.15\left(\mathrm{~m}, 2 \mathrm{H}, \mathrm{CH}_{2} \mathrm{lys}\right), 3.22\left(\mathrm{dd}, 1 \mathrm{H}, J_{\text {gem }}\right.$ $=15.0, J_{2}$, $\left.\mathrm{NH}=6.5 \mathrm{~Hz}, H-2^{\prime}\right), 3.46\left(\mathrm{dd}, 1 \mathrm{H}, J_{2}\right.$ ', NH $\left.=7.5 \mathrm{~Hz}, H-2^{\prime}\right), 3.74\left(\mathrm{~s}, 3 \mathrm{H}, \mathrm{OCH}_{3}\right), 3.84-$

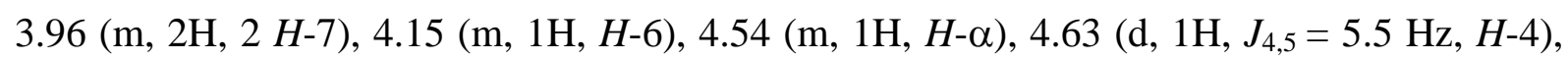
$4.70(\mathrm{t}, 1 \mathrm{H}, J=5.0 \mathrm{~Hz}, \mathrm{~N} H \mathrm{Boc}), 4.89\left(\mathrm{dd}, 1 \mathrm{H}, J_{5,6}=4.5 \mathrm{~Hz}, H-5\right), 5.08\left(\mathrm{~d}, 1 \mathrm{H}, J_{\text {gem }}=13.0\right.$ $\mathrm{Hz}, \mathrm{CH}-\mathrm{Ph}), 5.15$ (d, 1H, CH-Ph), 5.78 (m, 1H, NH Cbz), 6.98 (d, 1H, J= 7.5 Hz, NH), 7.337.41 (m, 5H, $5 \mathrm{H}$-Ar). ${ }^{13} \mathrm{C} \mathrm{NMR}\left(\mathrm{CDCl}_{3}, 62.9 \mathrm{MHz}\right): \delta=22.5\left(\mathrm{CH}_{2} \mathrm{lys}\right), 24.3\left(\mathrm{CH}_{3}\right), 25.8$ $\left(\mathrm{CH}_{3}\right), 28.4\left(\mathrm{C}_{(\mathrm{CH}}\right)_{3}$ boc $), 29.5\left(\mathrm{CH}_{2}\right.$ lys $), 31.8\left(\mathrm{CH}_{2}\right.$ lys $), 40.1,40.4\left(C-2, \mathrm{CH}_{2}\right.$ lys $), 42.9(C-$ 2'), 52.2, $52.5\left(C-\alpha, \mathrm{OCH}_{3}\right), 61.5(C-7), 66.9\left(C_{2}-\mathrm{Ph}\right), 79.4,81.7\left(C\left(\mathrm{CH}_{3}\right)_{3}\right.$ boc, $\left.C-5, C-6\right)$, 83.7, $84.9(C-3, C-4), 113.0\left(C\left(\mathrm{CH}_{3}\right)_{2}\right), 128.0$ (2 $C$-Ar), $128.1(C$-Ar), 128.5 (2 $C$-Ar), 136.4 ( $C$-Ar), 156.3, 156.9 ( $C=\mathrm{O}$ Boc, $C=\mathrm{O}$ Cbz), 170.9 ( $C=\mathrm{O}), 172.9$ ( $C=\mathrm{O}$ lys). ESI-HRMS $[\mathrm{M}+\mathrm{Na}]^{+} \mathrm{m} / \mathrm{z}=$ found 660.3112 (calculated for $\mathrm{C}_{31} \mathrm{H}_{47} \mathrm{~N}_{3} \mathrm{O}_{11} \mathrm{Na}:$ 660.3103). IR $(\mathrm{NaCl}) v_{\max } / \mathrm{cm}^{-1} 3345,2976,2933,2867,1709,1662,1525$

\section{Compound 18}

Compopund 14 was converted to amine 15 by catalytic hydrogenolysis (procedure C) and acid 17 was obtained by saponification of compound 16 (procedure B). Amine 15 (1.14 g, 2.2 mmol, 1.1 eq.) and acid 17 (1.28 g, $2 \mathrm{mmol}, 1$ eq.) were coupled according to procedure A to provide $18(1.53 \mathrm{~g}, 69 \%)$. White foam. $\mathrm{R} f=0.6(\mathrm{EA} / \mathrm{MeOH}: 9 / 1) .[\alpha]_{\mathrm{D}}=+1.6^{\circ}$ (c 0.2 in $\left.\mathrm{CHCl}_{3}\right) ;{ }^{1} \mathrm{H} \mathrm{NMR}\left(\mathrm{CDCl}_{3}, 400 \mathrm{MHz}\right): \delta=1.30-1.54$ (m, 8H, $4 \mathrm{CH}_{2}$ lys), 1.31 (s, 6H, $2 \mathrm{CH}_{3}$ ), $1.45\left(\mathrm{~s}, 18 \mathrm{H}, 2 \mathrm{C}\left(\mathrm{CH}_{3}\right)_{3}\right.$ boc), $1.48\left(\mathrm{~s}, 3 \mathrm{H}, \mathrm{CH}_{3}\right), 1.51\left(\mathrm{~s}, 3 \mathrm{H}, \mathrm{CH}_{3}\right), 1.63-1.86\left(\mathrm{~m}, 4 \mathrm{H}, 2 \mathrm{CH}_{2}\right.$ lys), 2.50-2.77 (m, 6H, 2 OH, $4 \mathrm{H}-2$ ), 3.03-3.18 (m, 5H, $2 \mathrm{CH}_{2}$ lys, $H-2$ '), 3.25 (dd, $1 \mathrm{H}, J_{\text {gem }}=$ $\left.15.0, J_{2}, \mathrm{NH}=5.5 \mathrm{~Hz}, H-2^{\prime}\right), 3.51\left(\mathrm{dd}, 1 \mathrm{H}, J_{\mathrm{gem}}=15.0, J_{2}\right.$ ', NH $=8.0 \mathrm{~Hz}, H-2$ ') $3.58(\mathrm{dd}, 1 \mathrm{H}$, $\left.J_{\text {gem }}=14.5, J_{2}, \mathrm{NH}=7.0 \mathrm{~Hz}, H-2^{\prime}\right), 3.75\left(\mathrm{~s}, 3 \mathrm{H}, \mathrm{OCH}_{3}\right), 3.82-3.88(\mathrm{~m}, 4 \mathrm{H}, 4 H-7), 4.13-4.18$ 
(m, 2H, $2 H-6), 4.31(\mathrm{~m}, 1 \mathrm{H}, H-\alpha), 4.41-4.48(\mathrm{~m}, 2 \mathrm{H}, H-\alpha, H-4), 4.69$ (d, $1 \mathrm{H}, J_{4,5}=5.5 \mathrm{~Hz}$, $H-4), 4.79-4.88$ (m, 4H, $2 H-5,2 \mathrm{NH}$ Boc), 5.07 (d, 1H, $\left.J_{\text {gem }}=13.0 \mathrm{~Hz}, \mathrm{CH}-\mathrm{Ph}\right), 5.16$ (d, 1H, $\mathrm{C} H-\mathrm{Ph}), 6.43$ (m, 1H, NH sucre), 7.15 (m, 1H, NH lys), 7.24 (m, 1H, NH lys), 7.31-7.41 (m, 6H, $5 \mathrm{H}$-Ar, $\mathrm{NH}$ sucre). ${ }^{13} \mathrm{C} \mathrm{NMR}\left(\mathrm{CDCl}_{3}, 100 \mathrm{MHz}\right): \delta=22.7,22.9$ (2 $\mathrm{CH}_{2}$ lys), 24.2, 24.3 (2 $\left.\mathrm{CH}_{3}\right), 25.8\left(2 \mathrm{CH}_{3}\right), 28.4\left(2 \mathrm{C}\left(\mathrm{CH}_{3}\right)_{3}\right), 29.5,29.6$ (2 $\mathrm{CH}_{2}$ lys), 30.8, 31.5 (2 $\mathrm{CH}_{2}$ lys), 39.6, 40.1, 40.7 (2 C-2, C-2', $2 C_{2}$ lys), 42.9 (C-2'), $52.7\left(C-\alpha, \mathrm{OCH}_{3}\right), 53.8(C-\alpha), 61.4,61.7$ (2 $C$-7), $66.8\left(\mathrm{CH}_{2}-\mathrm{Ph}\right), 79.2,79.3\left(2 \mathrm{C}\left(\mathrm{CH}_{3}\right)_{3}\right), 79.9$ (2 $C$-6), 81.2, 81.5 (2 C-5), 83.6, 84.0 (2 C4), 85.0 (2 C-3), 112.9 (2 $\left.C\left(\mathrm{CH}_{3}\right)_{2}\right), 128.0$ (2 $C$-Ar), 128.1 (C-Ar), 128.5 (2 C-Ar), 136.5 (CAr), 156.2 (2 $C=\mathrm{O}$ Boc), 157.3 ( $C=\mathrm{O}$ Cbz), 171.0, 171.6, 172.9, 173.9 (4 $C=\mathrm{O})$. ESI-HRMS $[\mathrm{M}+\mathrm{Na}]^{+} \mathrm{m} / \mathrm{z}=$ found 1131.5654 (calculated for $\mathrm{C}_{53} \mathrm{H}_{84} \mathrm{~N}_{6} \mathrm{O}_{19} \mathrm{Na}:$ 1131.5683). IR $(\mathrm{NaCl}) v_{\max } / \mathrm{cm}^{-1} 3336,2976,2933,2862,1692,1655,1525$

\section{Compound 19}

Compound 18 was deprotected by catalytic hydrogenation (procedure $\mathrm{C}$ ) and the corresponding amine (1.32 mg, $1.35 \mathrm{mmol}, 1.1$ eq.) was coupled with acid 17 (787 mg, 1.23 mmol, 1 eq.) according to procedure A to provide 19 (914 mg, 47\% yield). White solid. $\mathrm{Mp}=$ $117{ }^{\circ} \mathrm{C} . \mathrm{R} f=0.39(\mathrm{EA} / \mathrm{MeOH}: 9 / 1) .[\alpha]_{\mathrm{D}}=+1.2^{\circ}\left(\mathrm{c} 0.4\right.$ in $\left.\mathrm{CHCl}_{3}\right) .{ }^{1} \mathrm{H} \mathrm{NMR}\left(\mathrm{CDCl}_{3}, 400\right.$ MHz): $\delta=1.23-1.53\left(\mathrm{~m}, 12 \mathrm{H}, 6 \mathrm{CH}_{2}\right.$ lys), 1.28 (s, 3H, $\left.\mathrm{CH}_{3}\right), 1.31$ (s, $\left.6 \mathrm{H}, 2 \mathrm{CH}_{3}\right), 1.45$ (s, 27H, $3 \mathrm{C}\left(\mathrm{CH}_{3}\right)_{3}$ boc), 1.50 (s, 9H, $3 \mathrm{CH}_{3}$ ), 1.60-1.89 (m, 6H, $3 \mathrm{CH}_{2}$ lys), 2.42-2.60 (m, 3H, 3 $H-2$ ), 2.75-2.87 (m, 3H, $3 \mathrm{H}-2$ ), 3.03-3.19 (m, 7H, $3 \mathrm{CH}_{2}$ lys, $H-2$ '), 3.26 (dd, $1 \mathrm{H}, J_{\text {gem }}=14.0$, $J_{2}$, NH $\left.=4.0 \mathrm{~Hz}, H-2^{\prime}\right), 3.35\left(\mathrm{dd}, 1 \mathrm{H}, J_{\mathrm{gem}}=14.0, J_{2}\right.$, NH $=4.0 \mathrm{~Hz}, H-2$ '), 3.46-3.56 (m, 2H, 2 $H-2$ '), 3.65 (m, 1H, H-2'), 3.76 (s, 3H, $\left.\mathrm{OCH}_{3}\right), 3.80-3.90$ (m, 6H, $\left.6 H-7\right), 4.13-4.21$ (m, 4H, 3 $H-6, H-\alpha), 4.39-4.55$ (m, 5H, $2 H-\alpha, 2 H-4, \mathrm{~N} H$ Boc), 4.67 (d, 1H, $J_{4,5}=6.0$ Hz, H-4), 4.814.96 (m, 5H, $3 H-5,2 \mathrm{NH}$ Boc), 5.07 (d, 1H, J $\left.J_{\text {gem }}=12.0 \mathrm{~Hz}, \mathrm{CH}-\mathrm{Ph}\right), 5.15$ (d, 1H, CH-Ph), $6.47(\mathrm{~m}, 1 \mathrm{H}, \mathrm{NH}), 7.12(\mathrm{~m}, 1 \mathrm{H}, \mathrm{NH}), 7.31-7.43$ (m, 8H, $5 H$-Ar, $3 \mathrm{NH}), 7.93$ (m, 1H, NH). ${ }^{13} \mathrm{C} \mathrm{NMR}\left(\mathrm{CDCl}_{3}, 100 \mathrm{MHz}\right): \delta=22.7,22.8$ (3 $\mathrm{CH}_{2}$ lys), 24.3, $24.4\left(3 \mathrm{CH}_{3}\right), 25.8,25.9$ (3 $\mathrm{CH}_{3}$ ), 28.5 (3 $\left.\mathrm{C}\left(\mathrm{CH}_{3}\right)_{3}\right), 29.4,29.6,29.7$ (3 $\mathrm{CH}_{2}$ lys), 30.9, 31.2, 31.7 (3 $\mathrm{CH}_{2}$ lys), 39.6, 40.2, 40.3, 41.0, 42.7 (3 C-2, $3 C$-2', $3 C_{2}$ lys), 52.5, $52.7\left(C-\alpha, \mathrm{O} C H_{3}\right), 53.3(C-\alpha), 54.3(C-\alpha)$, 61.4, 61.7 (3 C-7), $66.8\left(\mathrm{CH}_{2}-\mathrm{Ph}\right), 79.1\left(3 \mathrm{C}\left(\mathrm{CH}_{3}\right)_{3}\right), 79.7,80.0,80.3$ (3 C-6), 81.3, 81.4, 81.6

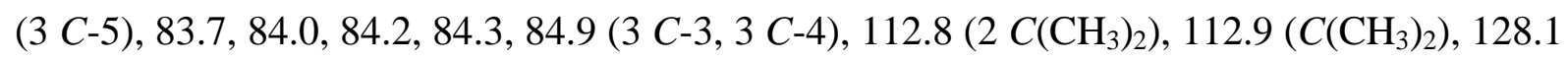
(2 $C$-Ar), 128.5 (3 $C$-Ar), 136.6 ( $C$-Ar), 156.2 (3 $C=\mathrm{O}$ Boc), 157.2 ( $C=\mathrm{O} \mathrm{Cbz}$ ), 170.8, 171.4, 171.6, 172.9, 173.2, 173.9 (6 C=O). ESI-HRMS $[\mathrm{M}+\mathrm{H}]^{+} \mathrm{m} / \mathrm{z}=$ found 1580.8481 (calculated for $\mathrm{C}_{75} \mathrm{H}_{122} \mathrm{~N}_{9} \mathrm{O}_{27}$ : 1580.8445). IR (NaCl) $\cup_{\max } / \mathrm{cm}^{-1}$ 3331, 2981, 2933, 2867, 1692, 1655, 1525 


\section{Compound 20}

Compound 19 was saponified (procedure B) and deprotected by catalytic hydrognolysis (procedure C). To a solution of deprotected glyco-amino acid (573 mg, $0.4 \mathrm{mmol}, 1 \mathrm{eq}$.) in $\mathrm{CH}_{2} \mathrm{Cl}_{2} / \mathrm{DMF}(4 / 1)$ at $0^{\circ} \mathrm{C}$ under argon, were added DIEA (346 $\mu \mathrm{L}, 2.0 \mathrm{mmol}, 5.0$ eq.) and HATU (182 mg, $0.48 \mathrm{mmol}, 1.2$ eq.). After stirring at room temperature for 3 days, the solvents were evaporated and the residue was taken up in ethyl acetate $(30 \mathrm{~mL})$. The solution was washed with brine $(10 \mathrm{~mL})$, dried over $\mathrm{MgSO}_{4}$, filtered and concentrated. The product was purified by column chromatography (silica gel, AcOEt/MeOH) to provide 20 (123 mg, $22 \%$ yield). White foam. $\mathrm{R} f=0.24(\mathrm{EtOAc} / \mathrm{MeOH}: 95 / 5)$. $[\alpha]_{\mathrm{D}}=-20.7^{\circ}\left(\mathrm{c} 0.2\right.$ in $\left.\mathrm{CHCl}_{3}\right) .{ }^{1} \mathrm{H}$ (MeOH-d 4,250 MHz): 1.27-1.50 (m, 4H, $2 \mathrm{CH}_{2}$ lys), 1.30 (s, 3H, $\left.\mathrm{CH}_{3}\right), 1.44$ (s, 9H, C(CH3) boc), 1.46 (s, 3H, $\left.\mathrm{CH}_{3}\right), 1.66\left(\mathrm{~m}, 1 \mathrm{H}, \mathrm{CH}_{2} \mathrm{lys}\right), 1.81$ (m, 1H, $\mathrm{CH}_{2}$ lys), 2.63 (d, 1H, $J_{\text {gem }}=$ $14.0 \mathrm{~Hz}, H-2), 2.69$ (d, 1H, H-2), 3.00-3.07 (m, 2H, CH $\mathrm{Cys}$ ), 3.29 (m, 1H, H-2'), 3.66-3.76 (m, 2H, H-2', H-7), $3.82\left(\mathrm{~m}, 1 \mathrm{H}, J_{\text {gem }}=11.0, J_{6,7}=4.5 \mathrm{~Hz}, H-7^{\prime}\right), 4.08$ (m, 1H, H-6), 4.26 $(\mathrm{m}, 1 \mathrm{H}, H-\alpha), 4.60\left(\mathrm{~d}, 1 \mathrm{H}, J_{4,5}=6.0 \mathrm{~Hz}, H-4\right), 4.87(\mathrm{~m}, 1 \mathrm{H}, H-5) .{ }^{13} \mathrm{C}\left(\mathrm{MeOH}_{-} \mathrm{d}_{4}, 62.9 \mathrm{MHz}\right)$ : 24.3, $24.7\left(\mathrm{CH}_{2}\right.$ lys, $\left.\mathrm{CH}_{3}\right), 26.3\left(\mathrm{CH}_{3}\right), 28.9\left(\mathrm{C}\left(\mathrm{CH}_{3}\right)_{3}\right), 30.6\left(\mathrm{CH}_{2}\right.$ lys $), 32.6\left(\mathrm{CH}_{2}\right.$ lys $), 39.6$ $(C-2), 41.1,41.2\left(C_{2}\right.$ lys, $C-2$ ') $, 55.2(\mathrm{C}-\alpha), 61.6(C-7), 79.9(C-6), 80.6\left(C\left(\mathrm{CH}_{3}\right)_{3}\right), 82.3(C-$ 5), $85.2(C-4), 85.9(C-3), 113.4\left(C\left(\mathrm{CH}_{3}\right)_{2}\right), 158.5$ ( $C=\mathrm{O}$ boc $), 173.0,175.1(2 C=\mathrm{O})$. ESIHRMS $[\mathrm{M}+\mathrm{Na}]^{+} \mathrm{m} / \mathrm{z}=$ found 1436.7626 (calculated for $\mathrm{C}_{66} \mathrm{H}_{111} \mathrm{~N}_{9} \mathrm{O}_{24} \mathrm{Na}$ : 1436.7634). IR $(\mathrm{NaCl}) \cup_{\max } / \mathrm{cm}^{-1} 3345,2976,2928,2862,1685,1657,1522,1454$

\section{Compound 21}

To a stirred solution of $8(42 \mathrm{mg}, 0.049 \mathrm{mmol})$ in $\mathrm{H}_{2} \mathrm{O}(0.50 \mathrm{ml})$ at $0^{\circ} \mathrm{C}$ was added trifluoroacetic acid $(0.50 \mathrm{ml})$. After completion of the reaction (TLC monitoring), the solvents were removed under reduced pressure and coevaporated with $\mathrm{Et}_{2} \mathrm{O}(3 \times 5 \mathrm{~mL})$. The residue was purified by column chromatography (RP-18 silica gel, $\mathrm{H}_{2} \mathrm{O} / \mathrm{MeOH}$ ) and lyophilized to provide 21 (35.6 $\mathrm{mg}, 99 \%$ yield).

White foam. $\mathrm{R} f=0.80\left(\mathrm{H}_{2} \mathrm{O} / \mathrm{MeOH}: 1 / 1\right) .[\alpha]_{\mathrm{D}}=+14.5^{\circ}\left(\mathrm{c} 0.4\right.$ in $\left.\mathrm{H}_{2} \mathrm{O}\right) .{ }^{1} \mathrm{H}\left(\mathrm{D}_{2} \mathrm{O}, 250 \mathrm{MHz}\right): \delta$ $=1.57\left(\mathrm{~d}, 3 \mathrm{H}, J=7.0 \mathrm{~Hz}, \mathrm{CH}_{3}\right.$ alanine $), 2.88\left(\mathrm{~d}, 1 \mathrm{H}, J_{\mathrm{gem}}=19.0 \mathrm{~Hz}, H-2\right), 3.04(\mathrm{~d}, 1 \mathrm{H}, H-2)$, $3.67\left(\mathrm{~s}, 2 \mathrm{H}, 2 H-2\right.$ ') $3.79\left(\mathrm{dd}, 1 \mathrm{H}, J_{\mathrm{gem}}=9.5, J_{5,6}=5.0 \mathrm{~Hz}, H-6\right), 4.13(\mathrm{q}, 1 \mathrm{H}, H-\alpha), 4.17$ (dd, $\left.1 \mathrm{H}, J_{5,6^{\prime}}=6.0 \mathrm{~Hz}, H-6^{\prime}\right), 4.54(\mathrm{~m}, 1 \mathrm{H}, H-5), 4.96\left(\mathrm{~d}, 1 \mathrm{H}, J_{4,5}=5.0 \mathrm{~Hz}, H-4\right) .{ }^{13} \mathrm{C}\left(\mathrm{D}_{2} \mathrm{O}, 62.9\right.$ MHz): $\delta=16.6\left(C_{3}\right.$ alanine $), 39.0,43.2(C-2, C-2 '), 49.1(C-\alpha), 70.3,70.4(C-6, C-5), 84.6$ (C-4), 87.2 (C-3), 114.0, 118.7 (TFA), 162.6, 163.2 (TFA), 171.4, 178.0 (2 $C=\mathrm{O})$. ESI-HRMS $[\mathrm{M}+\mathrm{H}]^{+} \mathrm{m} / \mathrm{z}=$ found 245.1165 (calculated for $\mathrm{C}_{10} \mathrm{H}_{17} \mathrm{~N}_{2} \mathrm{O}_{5}:$ 245.1132). IR (pellets) $\mathrm{U}_{\max } / \mathrm{cm}^{-1}$ $3383,3255,3094,2995,2938,1782,1678$ 


\section{Compound 22}

To a stirred solution of 25 (488 mg, $1.2 \mathrm{mmol})$ in methanol $(8 \mathrm{~mL})$ was added $\mathrm{HCl}(0.8 \mathrm{~mL})$ dropwise. After stirring at $50^{\circ} \mathrm{C}$ until completion of the reaction $(2 \mathrm{~h})$, the solvent was removed under reduced pressure. The residue was purified by column chromatography (silica gel, EA/MeOH) to provide $22\left(259 \mathrm{mg}, 82 \%\right.$ yield). White solide. $\mathrm{R} f=0.45$ (EA). $\mathrm{mp} 140^{\circ} \mathrm{C}$ (from diethyl ether). $[\alpha]_{\mathrm{D}}=-25.1^{\circ}\left(\mathrm{c} 0.1\right.$ in $\left.\mathrm{CH}_{3} \mathrm{OH}\right) .{ }^{1} \mathrm{H}$ NMR $\left(\mathrm{CD}_{3} \mathrm{OD}, 400 \mathrm{MHz}\right): \delta=2.93$ $\left(\mathrm{d}, 1 \mathrm{H}, J_{\text {gem }}=19.0 \mathrm{~Hz}, H-2\right), 3.00(\mathrm{~d}, 1 \mathrm{H}, H-2), 3.59\left(\mathrm{dd}, 1 \mathrm{H}, J_{\mathrm{gem}}=11.5 \mathrm{~Hz}, J_{8 \mathrm{a}-7}=6.0 \mathrm{~Hz}, H-\right.$ 8), $3.65\left(\mathrm{dd}, 1 \mathrm{H}, J_{8 \mathrm{~b}-7}=5.0 \mathrm{~Hz}, H-8\right), 3.96\left(\mathrm{app} \mathrm{td}, 1 \mathrm{H}, J_{7-6}=J_{7-8 \mathrm{~b}}=5.0 \mathrm{~Hz}, J_{7-8 \mathrm{a}}=6.0 \mathrm{~Hz}, \mathrm{H}-\right.$ 7), $4.07\left(\mathrm{dd}, 1 \mathrm{H}, J_{6-5}=4.0 \mathrm{~Hz}, H-6\right), 4.49\left(\mathrm{dd}, 1 \mathrm{H}, J_{5-4}=5.0 \mathrm{~Hz}, H-5\right), 4.88\left(\mathrm{~d}, 1 \mathrm{H}, J_{\text {gem }}=14.0\right.$ $\left.\mathrm{Hz}, H-2^{\prime}\right), 4.92$ (d, $\left.1 \mathrm{H}, H-2{ }^{\prime}\right), 5.14$ (d, $\left.1 \mathrm{H}, H-4\right) .{ }^{13} \mathrm{C}$ NMR $\left(\mathrm{CD}_{3} \mathrm{OD}, 100.6 \mathrm{MHz}\right): \delta=41.30$ (C-2), 64.0 (C-8), 72.0 (C-7), 72.4 (C-5), 78.9 (C-2'), 83.9 (C-6), 84.7 (C-3), 85.8 (C-4), $176.7(C=\mathrm{O})$. ESI-HRMS $[\mathrm{M}+\mathrm{Na}]^{+} \mathrm{m} / \mathrm{z}=$ found 286.0463 (calculated for $\mathrm{C}_{9} \mathrm{H}_{13} \mathrm{NNaO}_{8}$ : 286.0539). IR (NaCl) $U_{\max } / \mathrm{cm}^{-1} 3343,2949,1730,1647,11548,1436,1375,1207$

\section{Compound 24}

To a stirred solution of $11(503 \mathrm{mg}, 1.5 \mathrm{mmol})$ in $\mathrm{H}_{2} \mathrm{O}(3 \mathrm{~mL})$ was added TFA $(3 \mathrm{~mL})$ dropwise at $0^{\circ} \mathrm{C}$. After stirring at $\mathrm{rt}$ until completion of the reaction (6h), the solvent was removed under reduced pressure. The residue was co-evaporated with diethyl ether to provide 24 (438.4 mg, 99\% yield). Colourless gum. $\mathrm{R} f=0.40(\mathrm{MeOH} / \mathrm{EA}: 1 / 9)$. $[\alpha]_{\mathrm{D}}=+14.9^{\circ}(\mathrm{c} 0.1$ in $\left.\mathrm{CH}_{3} \mathrm{OH}\right) .{ }^{1} \mathrm{H}$ NMR $\left(\mathrm{CD}_{3} \mathrm{OD}, 400 \mathrm{MHz}\right): \delta=2.93\left(\mathrm{~d}, 1 \mathrm{H}, J_{\mathrm{gem}}=16.0 \mathrm{~Hz}, H-2\right), 2.98(\mathrm{~d}, 1 \mathrm{H}$, $H-2), 3.60(\mathrm{dd}, 1 \mathrm{H}, \mathrm{J}=11.0 \mathrm{~Hz}, \mathrm{~J}=5.5 \mathrm{~Hz}, \mathrm{H}-8), 3.66(\mathrm{dd}, 1 \mathrm{H}, \mathrm{J}=11.0 \mathrm{~Hz}, \mathrm{~J}=5.0 \mathrm{~Hz}, \mathrm{H}-$ 8),3.69 (s, 3H, $\left.\mathrm{OCH}_{3}\right), 3.91\left(\mathrm{app} \mathrm{td}, 1 \mathrm{H}, J_{7-8 \mathrm{a}}=J_{7-6}=5.5 \mathrm{~Hz}, J_{7-8 \mathrm{~b}}=5.0 \mathrm{~Hz}, H-7\right), 4.04$ (dd, $\left.1 \mathrm{H}, J_{6-7}=5.5 \mathrm{~Hz} ; J_{6-5}=4.5 \mathrm{~Hz}, H-6\right), 4.34\left(\mathrm{app} \mathrm{t}, 1 \mathrm{H}, J_{5-4}=J_{5-6}=4.5 \mathrm{~Hz}, H-5\right), 4.49(\mathrm{~d}, 1 \mathrm{H}$, $H-4), 4.75\left(\mathrm{~d}, 1 \mathrm{H}, J_{\text {gem }}=12.0 \mathrm{~Hz}, H-2^{\prime}\right), 5.10\left(\mathrm{~d}, 1 \mathrm{H}, H-2^{\prime}\right) .{ }^{13} \mathrm{C} \mathrm{NMR}\left(\mathrm{CD}_{3} \mathrm{OD}, 100.6 \mathrm{MHz}\right)$ $\delta=40.6(C-2), 52.2\left(C_{3}\right), 64.0(C-8), 72.3(C-7), 73.2(C-5), 78.1(C-4), 79.4(C-2)$ '), 81.8 $(C-3), \quad 82.1 \quad(C-6), \quad 172.3 \quad(C=\mathrm{O}) . \mathrm{IR} \quad(\mathrm{NaCl}) \quad \mathrm{U}_{\max } / \mathrm{cm}^{-}$ 3304, 2928, 1722, 1674, 1549, 1437, 1381, 1202.

\section{Compound 25}

Compound 10 was deprotected using general procedure B and the corresponding acide (1.30 g, $4.05 \mathrm{mmol}, 1$ eq.) was coupled with alanine methyl ester hydrochloride (905 mg, $1.6 \mathrm{eq}$, $6.48 \mathrm{mmol})$ following procedure A to provide $25(658 \mathrm{mg}, 40 \%$ yield). Yellow solide. $\mathrm{R} f=$ 0.55 (EA). mp $70^{\circ} \mathrm{C}$ (from diethyl ether). $[\alpha]_{\mathrm{D}}=-58.5^{\circ}$ (c 0.1 in $\left.\mathrm{CH}_{3} \mathrm{OH}\right) .{ }^{1} \mathrm{H}$ NMR $\left(\mathrm{CD}_{3} \mathrm{OD}\right.$, $400 \mathrm{MHz}): \delta=1.31\left(\mathrm{~s}, 3 \mathrm{H}, \mathrm{CH}_{3}\right), 1.38\left(\mathrm{~d}, 3 \mathrm{H}, J_{\mathrm{CH} 3 \mathrm{ala}-\mathrm{CHala}}=7.5 \mathrm{~Hz}, \mathrm{CH}_{3}\right.$ ala $), 1.47$ (s, $3 \mathrm{H}$, 
$\left.\mathrm{CH}_{3}\right), 2,82\left(\mathrm{~d}, 1 \mathrm{H}, J_{\mathrm{gem}}=16.0 \mathrm{~Hz}, H-2\right), 3.02(\mathrm{~d}, 1 \mathrm{H}, H-2), 3.64\left(\mathrm{dd}, 1 \mathrm{H}, J_{\text {gem }}=11.5 \mathrm{~Hz}, J_{8 \mathrm{a}-7}\right.$ $=5.5 \mathrm{~Hz}, H-8), 3.72\left(\mathrm{~s}, 3 \mathrm{H}, O_{C H}\right), 3.73\left(\mathrm{dd}, 1 \mathrm{H}, J_{8 \mathrm{~b}-7}=3.0 \mathrm{~Hz}, H-8\right), 3.91\left(\mathrm{ddd}, 1 \mathrm{H}, J_{7-6}=\right.$ $7.5 \mathrm{~Hz}, H-7), 4.01\left(\mathrm{dd}, 1 \mathrm{H}, J_{6-5}=3.5 \mathrm{~Hz}, H-6\right), 4.43$ (q, $1 \mathrm{H}, J_{\mathrm{Hala}-\mathrm{CH} 3 \mathrm{ala}}=7.5 \mathrm{~Hz}, H$-ala), 4.68 $\left(\mathrm{d}, 1 \mathrm{H}, J_{4-5}=6.0 \mathrm{~Hz}, H-4\right), 4.77\left(\mathrm{~d}, 1 \mathrm{H}, J_{\text {gem }}=12.0 \mathrm{~Hz}, H-2\right.$ '), 4.89 (dd, $\left.1 \mathrm{H}, H-5\right), 5.08(\mathrm{~d}$, 1H, $\left.\mathrm{H}-2^{\prime}\right) .{ }^{13} \mathrm{C}$ NMR (62.9 MHz, DMSO-D $): \delta=17.1\left(\mathrm{CH}_{3}\right.$ ala), $24.6\left(\mathrm{CH}_{3}\right), 25.9\left(\mathrm{CH}_{3}\right)$, $36.6(C-2), 47.5(C-\alpha), 50.1\left(\mathrm{OCH}_{3}\right), 63.1(C-8), 70.8(C-7), 75.8\left(C-2^{\prime}\right), 80.1(C-6), 80.7(C-$ 5), $82.8(C-3), 83.5(C-4), 111.5\left(C\left(\mathrm{CH}_{3}\right)_{2}\right), 169.3,173.4(2 \mathrm{C}=\mathrm{O})$. ESI-HRMS $[\mathrm{M}+\mathrm{Na}]^{+} \mathrm{m} / \mathrm{z}$ $=$ found 429.1502 (calculated for $\left.\mathrm{C}_{16} \mathrm{H}_{26} \mathrm{~N}_{2} \mathrm{NaO}_{10}: 429.1485\right)$. IR $(\mathrm{NaCl}) \mathrm{U}_{\max } / \mathrm{cm}^{-1}$ 3395, 2916, 1798, 1556, 1360, 1161.

\section{Acknowledgments}

This work was supported by The Région Lorraine and Institut Jean Barriol. The Ministère de l'Enseignement Supérieur et de la Recherche is acknowledged for $\mathrm{PhD}$ funding to $\mathrm{M}$.

Richard. We thank P. Lemière and F. Lachaud for technical assistance.

\section{Electronic supplementary information}

Copies of ${ }^{1} \mathrm{H}$ and ${ }^{13} \mathrm{C}$ NMR spectra, experimental procedures and characterizations of intermediates obtained from minor anomer 11.

\section{References}

1 D. J. Craik, D. P. Fairlie, S. Liras and D. Price, Chemi. Biol. Drug Des. 2013, 81, 136147.

2 T. Szekely, O. Roy, S. Faure and C. Taillefumier, Eur. J. Org. Chem., 2014, 2014, 56415657.

3 F. Giordanetto and J. Kihlberg, J. Med. Chem. 2014, 57, 278-295.

4 A. M. White and D. J. Craik, Expert Opin. Drug Discov., 2016, 11, 1151-1163.

5 C. Pifferi, N. Berthet and O. Renaudet, Biomater Sci., 2017, 5, 953-965.

6 M. A. T. Blaskovich, K. A. Hansford, M. S. Butler, Z. Jia, A. E. Mark and M. A. Cooper, ACS Infect. Dis., DOI:10.1021/acsinfecdis.7b00258.

7 D. A. Evans, C. J. Dinsmore, P. S. Watson, M. R. Wood, T. I. Richardson, B. W. Trotter and J. L. Katz, Angew. Chem. Int. Ed., 1998, 37, 2704-2708.

8 T. Mogi and K. Kita, Cell. Mol. Life Sci., 2009, 66, 3821-3826.

9 M. A. Abdalla, J. Nat. Med., 2016, 70, 708-720.

10 T. Velkov, K. D. Roberts and J. Li, Nat. Prod. Rep., 2017, 34, 295-309.

11 W. Bauer, U. Briner, W. Doepfner, R. Haller, R. Huguenin, P. Marbach, T. J. Petcher and null Pless, Life Sci., 1982, 31, 1133-1140.

12 R. Haubner, W. Schmitt, G. Hölzemann, S. L. Goodman, A. Jonczyk and H. Kessler, J. Am. Chem. Soc., 1996, 118, 7881-7891.

13 T. G. Kapp, F. Rechenmacher, S. Neubauer, O. V. Maltsev, E. A. Cavalcanti-Adam, R. Zarka, U. Reuning, J. Notni, H.-J. Wester, C. Mas-Moruno, J. Spatz, B. Geiger and H. Kessler, Sci. Rep., 2017, 7, 39805. 
14 R. Chapman, M. Danial, M. L. Koh, K. A. Jolliffe and S. Perrier, Chem. Soc. Rev., 2012, 41, 6023-6041.

15 N. Rodríguez-Vázquez, M. Amorín and J. R. Granja, Org. Biomol. Chem., 2017, 15, 4490-4505.

16 A. Tapeinou, M.-T. Matsoukas, C. Simal and T. Tselios, Biopolymers, 2015, 104, 453461.

17 D. J. Craik, J. E. Swedberg, J. S. Mylne and M. Cemazar, Expert Opin. Drug Disc., 2012, 7, 179-194.

18 A. M. Webster and S. L. Cobb, Chem. Eur J., DOI:10.1002/chem.201705340.

19 D. Arosio, L. Manzoni, C. Corno and P. Perego, Recent Pat. Anticancer Drug Discov., 2017, 12, 148-168.

20 E. Valeur, S. M. Guéret, H. Adihou, R. Gopalakrishnan, M. Lemurell, H. Waldmann, T. N. Grossmann and A. T. Plowright, Angew. Chem. Int. Ed., 2017, 56, 10294-10323.

21 R. Fransson, C. Sköld and A. Sandström, Strategies for conversion of peptides to peptidomimetic drugs, Ed by Vale, Nuno, in Biomedical Chemistry, 2015, 245-274.

22 S. H. Joo, Biomol. Ther., 2012, 20, 19-26.

23 E. Richard, C. Pifferi, M. Fiore, E. Samain, A. Le Gouëllec, S. Fort, O. Renaudet and B. Priem, ChemBioChem, 2017, 18, 1730-1734.

24 J. L. Jiménez Blanco, C. Ortiz Mellet and J. M. García Fernández, Chem. Soc. Rev., 2013, $42,4518-4531$.

25 G. C. Daskhan, N. Berthet, B. Thomas, M. Fiore and O. Renaudet, Carbohydr. Res., 2015, 405, 13-22.

26 P. Dumy, I. M. Eggleston, S. Cervigni, U. Sila, X. Sun and M. Mutter, Tetrahedron Lett., 1995, 36, 1255-1258.

27 M. Galibert, O. Renaudet, P. Dumy and D. Boturyn, Angew. Chem. Int. Ed., 2011, 50, 1901-1904.

28 I. Bossu, M. Šulc, K. Křenek, E. Dufour, J. Garcia, N. Berthet, P. Dumy, V. Křen and O. Renaudet, Org. Biomol. Chem., 2011, 9, 1948-1959.

29 M. Fiore, N. Berthet, A. Marra, E. Gillon, P. Dumy, A. Dondoni, A. Imberty and O. Renaudet, Org. Biomol. Chem., 2013, 11, 7113-7122.

30 J. W. Engle, H. Hong, Y. Zhang, H. F. Valdovinos, D. V. Myklejord, T. E. Barnhart, C. P. Theuer, R. J. Nickles and W. Cai, Mol. Pharm., 2012, 9, 1441-1448.

31 M. C. Galan, P. Dumy and O. Renaudet, Chem. Soc. Rev., 2013, 42, 4599-4612.

32 I. J. Krauss, J. G. Joyce, A. C. Finnefrock, H. C. Song, V. Y. Dudkin, X. Geng, J. D. Warren, M. Chastain, J. W. Shiver and S. J. Danishefsky, J. Am. Chem. Soc., 2007, 129, 11042-11044.

33 T. Ohta, N. Miura, N. Fujitani, F. Nakajima, K. Niikura, R. Sadamoto, C.-T. Guo, T. Suzuki, Y. Suzuki, K. Monde and S.-I. Nishimura, Angew. Chem. Int. Ed., 2003, 42, $5186-5189$.

34 O. Renaudet, D. Boturyn and P. Dumy, Synthesis and Biological Applications of Glycoconjugates, 2011, 129-144.

35 M. Richard, A.-S. Felten, C. Didierjean, M. Ruiz-Lopez, Y. Chapleur and N. PellegriniMoïse, Eur. J. Org. Chem., 2014, 33, 7364-7376.

36 N. Pellegrini-Moïse, M. Richard and Y. Chapleur, in Carbohydrate Chemistry, Ed A. Pilar Rauter, T. Lindhorst and Y. Queneau, Royal Society of Chemistry, Cambridge, 2014, vol. 40, pp. 99-117.

37 G. Enderlin, C. Taillefumier, C. Didierjean and Y. Chapleur, J. Org. Chem., 2009, 74, 8388-8391.

38 M. Andreini, C. Taillefumier, F. Chrétien, V. Thery and Y. Chapleur, J. Org. Chem., 2009, 74, 7651-7659. 
39 M. Richard, A.-S. Felten, F. Chrétien, M.-C. Averlant-Petit and N. Pellegrini-Moïse, Tetrahedron, 2017, 73, 6795-6804.

40 A.-S. Felten, N. Pellegrini-Moïse, K. Selmeczi, B. Henry and Y. Chapleur, Eur. J. Org. Chem., 2013, 25, 5645-5654.

41 V. Martí-Centelles, M. D. Pandey, M. I. Burguete and S. V. Luis, Chem. Rev., 2015, 115, 8736-8834.

42 V. Popsavin, S. Grabež, M. Popsavin, I. Krstić, V. Kojić, G. Bogdanović and V. Divjaković, Tetrahedron Lett., 2004, 45, 9409-9413.

43 A. Listkowski, O. Otman, S. Chambert and Y. Queneau, Tetrahedron Lett., 2015, 56, 5051-5053.

44 K. . Ravikumar and D. Farquhar, Tetrahedron Lett., 2002, 43, 1367-1368.

45 L. Schiavo, L. Jeanmart, S. Lanners, S. Choppin and G. Hanquet, New J. Chem., 2017, 41, 1421-1424. 